\title{
Modular Invariants from Subfactors: Type I Coupling Matrices and Intermediate Subfactors
}

\author{
Jens Böckenhauer and David E. Evans \\ School of Mathematics \\ University of Wales Cardiff \\ PO Box 926, Senghennydd Road \\ Cardiff CF24 4YH, Wales, U.K.
}

September 5, 2018

\begin{abstract}
A braided subfactor determines a coupling matrix $Z$ which commutes with the S- and T-matrices arising from the braiding. Such a coupling matrix is not necessarily of "type I", i.e. in general it does not have a block-diagonal structure which can be reinterpreted as the diagonal coupling matrix with respect to a suitable extension. We show that there are always two intermediate subfactors which correspond to left and right maximal extensions and which determine "parent" coupling matrices $Z^{ \pm}$of type I. Moreover it is shown that if the intermediate subfactors coincide, so that $Z^{+}=Z^{-}$, then $Z$ is related to $Z^{+}$by an automorphism of the extended fusion rules. The intertwining relations of chiral branching coefficients between original and extended S- and T-matrices are also clarified. None of our results depends on non-degeneracy of the braiding, i.e. the S- and T-matrices need not be modular. Examples from $S O(n)$ current algebra models illustrate that the parents can be different, $Z^{+} \neq Z^{-}$, and that $Z$ need not be related to a type I invariant by such an automorphism.
\end{abstract}

\section{Introduction}

A prominent problem in rational conformal field theory (RCFT) is the classification of modular invariants. Though it is usually a difficult task and solved only for a few special models (e.g. [8, 19]), its mathematical formulation is simple: For a given unitary, finite-dimensional representation of the modular group $S L(2 ; \mathbb{Z})$, let $S=$ $\left(S_{\lambda, \mu}\right)$ and $T=\left(T_{\lambda, \mu}\right)$ denote the matrices representing the generators $\left(\begin{array}{cc}0-1 \\ 1 & 0\end{array}\right)$ and $\left(\begin{array}{ll}1 & 1 \\ 0 & 1\end{array}\right)$, respectively. In the representations of interest $T$ is diagonal, $S$ is symmetric, $S^{2}$ is a permutation, and $S_{\lambda, 0} \geq S_{0,0}>0$. Here " 0 " is a distinguished label, referring to the "vacuum sector". A modular invariant is then a "coupling matrix" $Z$ (or "mass matrix") commuting with $S$ and $T$,

$$
S Z=Z S, \quad \text { and } \quad T Z=Z T,
$$


and subject to the constraints

$$
Z_{\lambda, \mu}=0,1,2, \ldots, \quad \text { and } \quad Z_{0,0}=1 .
$$

These constraints reflect the physical background of the problem: The coupling matrix usually describes multiplicities in the decomposition of the Hilbert space $\mathcal{H}_{\text {phys }}$ of physical states of a $2 \mathrm{D}$ conformal field theory under the action of a "symmetry algebra" $\mathcal{A} \otimes \mathcal{A}$ which is a tensor product of two copies" of a "chiral algebra" $\mathcal{A}$,

$$
\mathcal{H}_{\text {phys }}=\bigoplus_{\lambda, \mu} Z_{\lambda, \mu} H_{\lambda} \otimes H_{\mu}
$$

giving rise to a modular invariant partition function

$$
\mathcal{Z}=\sum_{\lambda, \mu} Z_{\lambda, \mu} \chi_{\lambda} \chi_{\mu}^{*}
$$

Here the $\chi_{\lambda}$ 's and $\chi_{\mu}$ 's are the conformal characters of the representations $H_{\lambda}$ and $H_{\mu}$, and the modular group action of $S$ and $T$ comes from resubstitution of their arguments, leaving the sesqui-linear combination $\mathcal{Z}$ invariant. The condition $Z_{0,0}=1$ then expresses the uniqueness of the vacuum state.

The simplest example for a coupling matrix is the "diagonal case", $Z_{\lambda, \mu}=\delta_{\lambda, \mu}$, which always gives a modular invariant partition function. More interesting nondiagonal modular invariants arise whenever the chiral algebra can be extended by some local fields. Of special relevance are the so-called type I invariants [11] for which the entries of the coupling matrices can be written as

$$
Z_{\lambda, \mu}=\sum_{\tau} b_{\tau, \lambda} b_{\tau, \mu}
$$

and which refer directly to the extension through their "block-diagonal" structure: The label $\tau$ runs over the representations of the extended chiral algebra $\mathcal{A}^{\text {ext }}$, and the non-negative integers $b_{\tau, \lambda}$ describe the branching of a representation $\tau$ into $\lambda$ 's according to the inclusion $\mathcal{A} \subset \mathcal{A}^{\text {ext }}$. The branching coefficients fulfill $b_{\tau, 0}=\delta_{\tau, 0}$ (by some abuse of notation we denote the vacuum sector of $\mathcal{A}^{\text {ext }}$ also by " 0 "), thus guaranteeing the normalization condition $Z_{0,0}=1$. Rewriting the partition function in terms of the extended characters $\chi_{\tau}^{\text {ext }}=\sum_{\lambda} b_{\tau, \lambda} \chi_{\lambda}$, any type I modular invariant can be considered as completely diagonal: $Z_{\tau, \tau^{\prime}}^{\text {ext }}=\delta_{\tau^{\prime}, \tau}$. It is argued in [12, 29, that after extending the chiral algebras maximally, the coupling matrix of a partition function in RCFT is at most a permutation, $Z_{\tau, \tau^{\prime}}^{\text {ext }}=\delta_{\tau^{\prime}, \omega(\tau)}$, where the permutation $\omega$ is an automorphism of the extended fusion rules, satisfying $\omega(0)=0$. As a consequence, a maximal extension $\mathcal{A} \subset \mathcal{A}^{\text {ext }}$ in RCFT produces a coupling matrix of some modular invariant partition function which can be written as

$$
Z_{\lambda, \mu}=\sum_{\tau} b_{\tau, \lambda} b_{\omega(\tau), \mu}
$$

\footnotetext{
${ }^{1}$ In generic RCFTs one does not necessarily start with a symmetry algebra made of two identical chiral algebras. However, Eq. (1) is designed for such a symmetric situation, whereas in the "heterotic" situation one has to deal with different S- and T-matrices intertwined by Z.
} 
Partition functions of the form Eq. (㺼) which are not of type I, Eq. (3), are usually referred to as being "type II" [11.

Given matrices $S$ and $T$ arising from the modular transformations of a collection of characters $\chi_{\lambda}$ in a RCFT, the solution of the mathematical problem given in Eqs. (11) and (2) can neverthelss yield coupling matrices which are neither of type I nor of type II. Note that a coupling matrix of the form in Eq. (雨) has necessarily "symmetric vacuum coupling", $Z_{\lambda, 0}=Z_{0, \lambda}$. However, even for rather well-behaved models like $S O(n)$ current algebras there are known matrices $Z$ satisfying Eqs. (11) and (2), but which do not have this symmetry, cf. Section 7 below. Chiral algebras often admit different extensions, and only then, but much more rarely, modular invariants without symmetric vacuum coupling have been found. Namely, it can happen that two chiral extensions $\mathcal{A} \subset \mathcal{A}_{ \pm}^{\text {ext }}$ of the original chiral algebra $\mathcal{A}$ are compatible such that a given coupling matrix has to be interpreted as an "automorphism" invariant with respect to the enhanced "heterotic" symmetry algebra $\mathcal{A}_{+}^{\text {ext }} \otimes \mathcal{A}_{-}^{\text {ext }}$. (It seems that this possibility has sometimes been ignored in the literature although the heterotic case was taken into account in [29].) Unfortunately the standard terminology "permutation" and "automorphism" is a bit misleading in the heterotic case because the labels of left and right sectors are generically different. A more precise notion would be "bijection" and "isomorphism of fusion rules", and the distinction between diagonal and permutation invariant does no longer make sense for a maximally extended heterotic symmetry algebra. Finally, in case that for a fixed theory there are several modular invariant partition functions it may happen that a linear combination of their coupling matrices yields a solution of Eq. (2), which may however fail to have a consistent interpretation as a partition function [37, 39, 18]. Such modular invariants without physical interpretation seem to be extremely rare.

The mathematical classification problem of Eqs. (11) and (2) was considered in [6, [] by means of subfactor theory, using the ideas of $\alpha$-induction [28, 41, 3, 4, 5] and double triangle algebras [30]. The analysis in [6, [] addressed in particular the problem of understanding the relation between modular invariants, graphs and "nimreps" (non-negative integer valued matrix representations of the Verlinde fusion algebra) - a puzzling connection going back to the celebrated A-D-E classification of [8, 26], its general nature noticed in [10, 11] and further studied in [9, 31, 1]. It follows from [32, 16, 15] that a (type III) von Neumann factor $N$ with a system ${ }_{N} \mathcal{X}_{N}$ of braided endomorphisms give rise to certain "statistics" matrices $S$ and $T$, which are modular whenever the braiding is non-degenerate [32]. It was shown in [6] that then an inclusion $N \subset M$ of von Neumann factors which is compatible with the system ${ }_{N} \mathcal{X}_{N}$ determines a coupling matrix $Z$ by $\alpha$-induction,

$$
Z_{\lambda, \mu}=\left\langle\alpha_{\lambda}^{+}, \alpha_{\mu}^{-}\right\rangle, \quad \lambda, \mu \in{ }_{N} \mathcal{X}_{N},
$$

solving Eqs. (1) and (2) even if the braiding is degenerate. Here $\alpha_{\lambda}^{+}$and $\alpha_{\mu}^{-}$are the two inductions of $\lambda$ and $\mu$, coming from braiding and opposite braiding, and the bracket $\left\langle\alpha_{\lambda}^{+}, \alpha_{\mu}^{-}\right\rangle$denotes the dimension of their relative intertwiner space. From current algebra models ("WZW") in RCFT one can construct braided subfactors such that the statistics matrices $S$ and $T$ and the Kac-Peterson matrices performing the 
$S L(2 ; \mathbb{Z})$ transformations of the affine characters (cf. [25]) coincide. This connection between statistics and conformal character transformations is expected to hold quite generally in RCFT (e.g. it was conjectured in [17]), and the conformal spin-statistics theorem [16, 15, 20] establishes this for the T-matrices. To prove this for the Smatrices requires one to show that the composition of superselection sectors indeed recovers the Verlinde fusion rules, and this has been done for several models, most significantly for $S U(n)$ at all levels in [40]. For local extensions (cf. [36]) the subfactor $N \subset M$ can be thought of as a version of the inclusion $\mathcal{A} \subset \mathcal{A}^{\text {ext }}$. In terms of (a variation of) the $\alpha$-induction formula of [28], such subfactors were first investigated for certain conformal inclusions in [41]. This was further analyzed and extended to simple

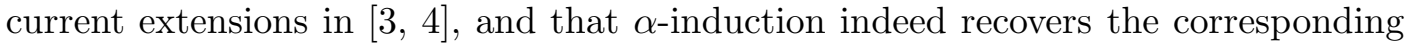
modular invariants was found (for $S U(2)$ and $S U(3)$ current algebras) in [5].

The two inductions, $\alpha^{+}$and $\alpha^{-}$, produce chiral systems ${ }_{M} \mathcal{X}_{M}^{+}$and ${ }_{M} \mathcal{X}_{M}^{-}$, intersecting on the "ambichiral" system ${ }_{M} \mathcal{X}_{M}^{0}$. Then Eq. (5) can be written as

$$
Z_{\lambda, \mu}=\sum_{\tau \in_{M} \mathcal{X}_{M}^{0}} b_{\tau, \lambda}^{+} b_{\tau, \mu}^{-}
$$

with chiral branching coefficients $b_{\tau, \lambda}^{ \pm}=\left\langle\tau, \alpha_{\lambda}^{ \pm}\right\rangle$. Now the question arises whether the general subfactor setting of [6, 7], which is also able to produce type II invariants so that in particular $b_{\tau, \lambda}^{+} \neq b_{\tau, \lambda}^{-}$is possible, will be confined to coupling matrices of the form of Eq. (牙) or whether it can even produce other solutions of Eqs. (11) and (2), e.g. with heterotic vacuum coupling. This is the issue of the present paper. We will indeed demonstrate that our framework incorporates the general situation, including modular invariants corresponding to heterotic extensions of the symmetry algebra.

In fact, we study subfactors $N \subset M$, producing coupling matrices $Z$, through intermediate subfactors, making essential use of the Galois correspondence elaborated in [23]. We derive in Section 4 that there are intermediate subfactors $M_{+}$and $M_{-}$, $N \subset M_{ \pm} \subset M$, naturally associated to the vacuum column $\left(Z_{\lambda, 0}\right)$ respectively the vacuum row $\left(Z_{0, \lambda}\right)$ of the coupling matrix determined by $N \subset M$. The subfactors $N \subset M_{ \pm}$in turn determine coupling matrices $Z^{ \pm}$which are of the form of Eq. (3) and can be interpreted as the "type I parents" of the original coupling matrix $Z$. In Section 5 we show that in the case $M_{+}=M_{-}$, so that in particular there is a unique parent $Z^{+}=Z^{-}$, the coupling matrix is indeed of the form of Eq. (曾), recovering a fusion rule automorphism of the ambichiral system. For the general situation we prove a proposition which shows that $M_{+}$and $M_{-}$should be regarded as the operator algebraic version of maximally extended left and right chiral algebras, using a recent result of Rehren [35]. In Section 6 we establish the intertwining relations of the chiral branching coefficients between the original S- and T-matrices and the "extended" ones arising from the ambichiral braiding. It is remarkable that the entire analysis does not need to assume that the braiding is non-degenrate, i.e. our results remain valid even if the matrices $S$ and $T$ are not modular. In Section 7 we finally show by examples from $S O(n)$ current algebras that indeed $M_{+} \neq M_{-}$is possible, that the parents can be different, $Z^{+} \neq Z^{-}$, and that subfactors can produce coupling matrices $Z$ which have heterotic vacuum coupling, so that Eq. (荬) can not be adopted in general. 


\section{Preliminaries}

Let $A$ and $B$ be type III von Neumann factors. A unital $*$-homomorphism $\rho: A \rightarrow B$ is called a $B$-A morphism. The positive number $d_{\rho}=[B: \rho(A)]^{1 / 2}$ is called the statistical dimension of $\rho$; here $[B: \rho(A)]$ is the Jones index 24] of the subfactor $\rho(A) \subset B$. If $\rho$ and $\sigma$ are $B-A$ morphisms with finite statistical dimensions, then the vector space of intertwiners

$$
\operatorname{Hom}(\rho, \sigma)=\{t \in B: t \rho(a)=\sigma(a) t, a \in A\}
$$

is finite-dimensional, and we denote its dimension by $\langle\rho, \sigma\rangle$. An $A$ - $B$ morphism $\bar{\rho}$ is a conjugate morphism if there are isometries $r_{\rho} \in \operatorname{Hom}\left(\mathrm{id}_{A}, \bar{\rho} \rho\right)$ and $\bar{r}_{\rho} \in \operatorname{Hom}\left(\operatorname{id}_{B}, \rho \bar{\rho}\right)$ such that $\rho\left(r_{\rho}\right)^{*} \bar{r}_{\rho}=d_{\rho}^{-1} \mathbf{1}_{B}$ and $\bar{\rho}\left(\bar{r}_{\rho}\right)^{*} r_{\rho}=d_{\rho}^{-1} \mathbf{1}_{A}$. The map $\phi_{\rho}: B \rightarrow A, b \mapsto$ $r_{\rho}^{*} \bar{\rho}(b) r_{\rho}$, is called the (unique) standard left inverse and satisfies

$$
\phi_{\rho}\left(\rho(a) b \rho\left(a^{\prime}\right)\right)=a \phi_{\rho}(b) a^{\prime}, \quad a, a^{\prime} \in A, \quad b \in B .
$$

If $t \in \operatorname{Hom}(\rho, \sigma)$ then we have

$$
d_{\rho} \phi_{\rho}(b t)=d_{\sigma} \phi_{\sigma}(t b), \quad b \in B .
$$

We work with the setting of [6], i.e. we are working with a type III subfactor and finite system ${ }_{N} \mathcal{X}_{N} \subset \operatorname{End}(N)$ of (possibly degenerately) braided morphisms which is compatible with the inclusion $N \subset M$. Then the inclusion is in particular forced to have finite Jones index and also finite depth (see e.g. [14]). More precisely, we make the following

Assumption 2.1 We assume that we have a type III subfactor $N \subset M$ together with a finite system of endomorphisms ${ }_{N} \mathcal{X}_{N} \subset \operatorname{End}(N)$ in the sense of [6, Def. 2.1] which is braided in the sense of [6, Def. 2.2] and such that $\theta=\bar{\iota} \iota \in \Sigma\left({ }_{N} \mathcal{X}_{N}\right)$ for the injection $M-N$ morphism $\iota: N \hookrightarrow M$ and a conjugate $N$ - $M$ morphism $\bar{\iota}$.

With the braiding $\varepsilon$ on ${ }_{N} \mathcal{X}_{N}$ and its extension to $\Sigma\left({ }_{N} \mathcal{X}_{N}\right)$ (the set of finite sums of morphisms in ${ }_{N} \mathcal{X}_{N}$ ) as in [6], one can define the $\alpha$-induced morphisms $\alpha_{\lambda}^{ \pm} \in \operatorname{End}(M)$ for $\lambda \in \Sigma\left({ }_{N} \mathcal{X}_{N}\right)$ by the Longo-Rehren formula [28], namely by putting

$$
\alpha_{\lambda}^{ \pm}=\bar{\iota}^{-1} \circ \operatorname{Ad}\left(\varepsilon^{ \pm}(\lambda, \theta)\right) \circ \lambda \circ \bar{\iota},
$$

where $\bar{\iota}$ denotes a conjugate morphism of the injection map $\iota: N \hookrightarrow M$. Then $\alpha_{\lambda}^{+}$and $\alpha_{\lambda}^{-}$extend $\lambda$, i.e. $\alpha_{\lambda}^{ \pm} \circ \iota=\iota \circ \lambda$, which in turn implies $d_{\alpha_{\lambda}^{ \pm}}=d_{\lambda}$ by the multiplicativity of the minimal index [27]. Let $\gamma=\iota \bar{\imath}$ denote Longo's canonical endomorphism from $M$ into $N$. Then there is an isometry $v \in \operatorname{Hom}(\mathrm{id}, \gamma)$ such that any $m \in M$ is uniquely decomposed as $m=n v$ with $n \in N$. Thus the action of the extensions $\alpha_{\lambda}^{ \pm}$ are uniquely characterized by the relation $\alpha_{\lambda}^{ \pm}(v)=\varepsilon^{ \pm}(\lambda, \theta)^{*} v$ which can be derived from the braiding fusion equations (BFE's, see e.g. [6, Eq. (5)]). Moreover, we have $\alpha_{\lambda \mu}^{ \pm}=\alpha_{\lambda}^{ \pm} \alpha_{\mu}^{ \pm}$if also $\mu \in \Sigma\left({ }_{N} \mathcal{X}_{N}\right)$, and clearly $\alpha_{\operatorname{id}_{N}}^{ \pm}=\operatorname{id}_{M}$. In general one has

$$
\operatorname{Hom}(\lambda, \mu) \subset \operatorname{Hom}\left(\alpha_{\lambda}^{ \pm}, \alpha_{\mu}^{ \pm}\right) \subset \operatorname{Hom}(\iota \lambda, \iota \mu), \quad \lambda, \mu \in \Sigma\left({ }_{N} \mathcal{X}_{N}\right) .
$$


The first inclusion is a consequence of the BFE's. Namely, $t \in \operatorname{Hom}(\lambda, \mu)$ obeys $t \varepsilon^{ \pm}(\theta, \lambda)=\varepsilon^{ \pm}(\theta, \mu) \theta(t)$, and thus

$$
t \alpha_{\lambda}^{ \pm}(v)=t \varepsilon^{ \pm}(\lambda, \theta)^{*} v=\varepsilon^{ \pm}(\mu, \theta)^{*} \theta(t) v=\varepsilon^{ \pm}(\mu, \theta)^{*} v t=\alpha_{\mu}^{ \pm}(v) t .
$$

The second follows from the extension property of $\alpha$-induction. Hence $\alpha_{\bar{\lambda}}^{ \pm}$is a conjugate for $\alpha_{\lambda}^{ \pm}$as there are $r_{\lambda} \in \operatorname{Hom}(\mathrm{id}, \bar{\lambda} \lambda) \subset \operatorname{Hom}\left(\mathrm{id}, \alpha_{\bar{\lambda}}^{ \pm} \alpha_{\lambda}^{ \pm}\right)$and $\bar{r}_{\lambda} \in \operatorname{Hom}(\mathrm{id}, \lambda \bar{\lambda}) \subset$ $\operatorname{Hom}\left(\mathrm{id}, \alpha_{\lambda}^{ \pm} \alpha_{\bar{\lambda}}^{ \pm}\right)$such that $\lambda\left(r_{\lambda}\right)^{*} \bar{r}_{\lambda}=\bar{\lambda}\left(\bar{r}_{\lambda}\right)^{*} r_{\lambda}=d_{\lambda}^{-1} \mathbf{1}$. We also have some kind of naturality equations for $\alpha$-induced morphisms,

$$
x \varepsilon^{ \pm}(\rho, \lambda)=\varepsilon^{ \pm}(\rho, \mu) \alpha_{\rho}^{ \pm}(x)
$$

whenever $x \in \operatorname{Hom}(\iota \lambda, \iota \mu)$ and $\rho \in \Sigma\left({ }_{N} \mathcal{X}_{N}\right)$.

Recall that the statistics phase of $\omega_{\lambda}$ for $\lambda \in{ }_{N} \mathcal{X}_{N}$ is given as

$$
d_{\lambda} \phi_{\lambda}\left(\varepsilon^{+}(\lambda, \lambda)\right)=\omega_{\lambda} \mathbf{1}
$$

The monodromy matrix $Y$ is defined by

$$
Y_{\lambda, \mu}=\sum_{\rho \in \in_{N} \mathcal{X}_{N}} \frac{\omega_{\lambda} \omega_{\mu}}{\omega_{\rho}} N_{\lambda, \mu}^{\rho} d_{\rho}, \quad \lambda, \mu \in{ }_{N} \mathcal{X}_{N},
$$

with $N_{\lambda, \mu}^{\rho}=\langle\rho, \lambda \mu\rangle$ denoting the fusion coefficients. Then one checks that $Y$ is symmetric, that $Y_{\bar{\lambda}, \mu}=Y_{\lambda, \mu}^{*}$ as well as $Y_{\lambda, 0}=d_{\lambda}$ [32, 16, 15]. (As usual, the label "0" refers to the identity morphism id $\in{ }_{N} \mathcal{X}_{N}$.) Now let $\Omega$ be the diagonal matrix with entries $\Omega_{\lambda, \mu}=\omega_{\lambda} \delta_{\lambda, \mu}$. Putting $Z_{\lambda, \mu}=\left\langle\alpha_{\lambda}^{+}, \alpha_{\mu}^{-}\right\rangle$defines a matrix subject to the constraints Eq. (2) and commuting with $Y$ and $\Omega$ [6]. The $\mathrm{Y}$ - and $\Omega$-matrices obey $\Omega Y \Omega Y \Omega=z Y$ where $z=\sum_{\lambda} d_{\lambda}^{2} \omega_{\lambda}[32,16,15]$, and this actually holds even if the braiding is degenerate (see [6, Sect. 2]). If $z \neq 0$ we put $c=4 \arg (z) / \pi$, which is defined modulo 8, and call it the "central charge". Moreover, S- and T-matrices are then defined by

$$
S=|z|^{-1} Y, \quad T=\mathrm{e}^{-\mathrm{i} \pi c / 12} \Omega
$$

and hence fulfill TSTST $=S$. One has $|z|^{2}=w$ with the global index $w=\sum_{\lambda} d_{\lambda}^{2}$ and $S$ is unitary, so that $S$ and $T$ are indeed the standard generators in a unitary representation of the modular group $S L(2 ; \mathbb{Z})$, if and only if the braiding is nondegenerate [32]. Consequently, $Z$ gives a modular invariant in this case.

Let ${ }_{M} \mathcal{X}_{M} \subset \operatorname{End}(M)$ denote a system of endomorphisms consisting of a choice of representative endomorphisms of each irreducible subsector of sectors of the form $[\iota \lambda \bar{\iota}], \lambda \in{ }_{N} \mathcal{X}_{N}$. We choose id $\in \operatorname{End}(M)$ representing the trivial sector in ${ }_{M} \mathcal{X}_{M}$. Then we define similarly the chiral systems ${ }_{M} \mathcal{X}_{M}^{ \pm}$and the $\alpha$-system ${ }_{M} \mathcal{X}_{M}^{\alpha}$ to be the subsystems of endomorphisms $\beta \in{ }_{M} \mathcal{X}_{M}$ such that $[\beta]$ is a subsector of $\left[\alpha_{\lambda}^{ \pm}\right]$and of of $\left[\alpha_{\lambda}^{+} \alpha_{\mu}^{-}\right]$, respectively, for some $\lambda, \mu \in{ }_{N} \mathcal{X}_{N}$. (Note that any subsector of $\left[\alpha_{\lambda}^{+} \alpha_{\mu}^{-}\right]$is automatically a subsector of $[\iota \nu \bar{\iota}]$ for some $\nu \in{ }_{N} \mathcal{X}_{N}$.) The ambichiral system is defined as the intersection ${ }_{M} \mathcal{X}_{M}^{0}={ }_{M} \mathcal{X}_{M}^{+} \cap{ }_{M} \mathcal{X}_{M}^{-}$, so that ${ }_{M} \mathcal{X}_{M}^{0} \subset{ }_{M} \mathcal{X}_{M}^{ \pm} \subset{ }_{M} \mathcal{X}_{M}^{\alpha} \subset{ }_{M} \mathcal{X}_{M}$. Their "global indices", i.e. their sums over the squares of the statistical dimensions are denoted by $w_{0}, w_{ \pm}, w_{\alpha}$ and $w$, and thus fulfill $1 \leq w_{0} \leq w_{ \pm} \leq w_{\alpha} \leq w$. 


\section{More on global indices and chiral locality}

Recall from [07, Prop. 3.1] that $w_{+}=w_{-}$and that $w / w_{+}=\sum_{\lambda \in_{N} \mathcal{X}_{N}} d_{\lambda} Z_{\lambda, 0}$. We will now derive a general formula for the $\alpha$-global index $w_{\alpha}=\sum_{\beta \in_{M}} \mathcal{X}_{M}^{\alpha} d_{\beta}^{2}$ and also for $w_{0}$. We denote by ${ }_{N} \mathcal{X}_{N}^{\mathrm{deg}} \subset{ }_{N} \mathcal{X}_{N}$ the subsystem of degenerate morphisms.

Proposition 3.1 The $\alpha$-global index is given by

$$
w_{\alpha}=\frac{w}{\sum_{\lambda \in \epsilon_{N}} \mathcal{X}_{N}^{\operatorname{deg}} Z_{0, \lambda} d_{\lambda}} .
$$

Moreover, the ambichiral global index is given by $w_{0}=w_{+}^{2} / w_{\alpha}$.

Proof. Let $R_{\lambda, \mu}, \lambda, \mu \in{ }_{N} \mathcal{X}_{N}$, denote matrices with entries $R_{\lambda, \mu ; \beta}^{\beta^{\prime}}=\left\langle\beta \alpha_{\lambda}^{+} \alpha_{\mu}^{-}, \beta^{\prime}\right\rangle$, $\beta, \beta^{\prime} \in{ }_{M} \mathcal{X}_{M}^{\alpha}$. Further let $\vec{d}$ denote the column vector with entries $d_{\beta}, \beta \in{ }_{M} \mathcal{X}_{M}^{\alpha}$. Then $\vec{d}$ is a simultaneous eigenvector of the matrices $R_{\lambda, \mu}$ with respective eigenvalues $d_{\lambda} d_{\mu}$. We define another vector $\vec{v}$ by putting

$$
v_{\beta}=\sum_{\lambda, \mu \in \in_{N} \mathcal{X}_{N}} d_{\lambda} d_{\mu}\left\langle\beta, \alpha_{\lambda}^{+} \alpha_{\mu}^{-}\right\rangle, \quad \beta \in{ }_{M} \mathcal{X}_{M}^{\alpha}
$$

Then we have $R_{\lambda, \mu} \vec{v}=d_{\lambda} d_{\mu} \vec{v}$, as we can compute

$$
\begin{aligned}
\left(R_{\lambda, \mu} \vec{v}\right)_{\beta} & =\sum_{\beta^{\prime} \in_{M} \mathcal{X}_{M}^{\alpha}} \sum_{\nu, \rho \in N} \mathcal{X}_{N}\left\langle\beta \alpha_{\lambda}^{+} \alpha_{\mu}^{-}, \beta^{\prime}\right\rangle d_{\nu} d_{\rho}\left\langle\beta^{\prime}, \alpha_{\nu}^{+} \alpha_{\rho}^{-}\right\rangle \\
& =\sum_{\nu, \rho \in_{N} \mathcal{X}_{N}} d_{\nu} d_{\rho}\left\langle\beta \alpha_{\lambda}^{+} \alpha_{\mu}^{-}, \alpha_{\nu}^{+} \alpha_{\rho}^{-}\right\rangle \\
& =\sum_{\nu, \rho, \xi, \eta \in_{N}} \mathcal{X}_{N} d_{\nu} d_{\rho} N_{\nu, \bar{\lambda}}^{\xi} N_{\rho, \bar{\mu}}^{\eta}\left\langle\beta, \alpha_{\xi}^{+} \alpha_{\eta}^{-}\right\rangle \\
& =\sum_{\xi, \eta \in_{N} \mathcal{X}_{N}} d_{\lambda} d_{\mu} d_{\xi} d_{\eta}\left\langle\beta, \alpha_{\xi}^{+} \alpha_{\eta}^{-}\right\rangle=d_{\lambda} d_{\mu} v_{\beta} .
\end{aligned}
$$

Because the sum matrix $\sum_{\lambda, \mu} R_{\lambda, \mu}$ is irreducible it follows $\vec{v}=\zeta \vec{d}, \zeta \in \mathbb{R}$, by the uniqueness of the Perron-Frobenius eigenvector. Note that $d_{\lambda} d_{\mu}=\sum_{\beta}\left\langle\beta, \alpha_{\lambda}^{+} \alpha_{\mu}^{-}\right\rangle d_{\beta}$, and hence $w^{2}=\sum_{\beta} v_{\beta} d_{\beta}=\zeta w_{\alpha}$. We next notice that $\zeta=v_{0}$ as $d_{0}=1$. But $v_{0}$ can be computed as

$$
v_{0}=\sum_{\lambda, \mu \in \in_{N} \mathcal{X}_{N}} d_{\lambda} d_{\mu}\left\langle\alpha_{\bar{\lambda}}^{+}, \alpha_{\mu}^{-}\right\rangle=\sum_{\lambda, \mu \in \in_{N} \mathcal{X}_{N}} Y_{0, \lambda} Z_{\lambda, \mu} Y_{\mu, 0}=\sum_{\lambda, \mu \in \in_{N} \mathcal{X}_{N}} Z_{0, \lambda} Y_{\lambda, \mu} Y_{\mu, 0}
$$

where we used commutativity of the monodromy matrix $Y$ with the coupling matrix $Z$ [6, Thm. 5.7]. By Rehren's argument [32] we have

$$
\sum_{\mu \in \in_{N} \mathcal{X}_{N}} Y_{\lambda, \mu} Y_{\mu, 0}=\left\{\begin{array}{cl}
w d_{\lambda} & \lambda \in{ }_{N} \mathcal{X}_{N}^{\mathrm{deg}} \\
0 & \lambda \notin{ }_{N} \mathcal{X}_{N}^{\mathrm{deg}}
\end{array} .\right.
$$

Hence $\zeta=w \sum_{\lambda \in_{N}} \mathcal{X}_{N}^{\operatorname{deg}} Z_{0, \lambda} d_{\lambda}$, establishing Eq. (10). 
Next we define two vectors $\vec{v}^{ \pm}$with entries $v_{\beta}^{ \pm}=\sum_{\lambda} d_{\lambda}\left\langle\beta, \alpha_{\lambda}^{ \pm}\right\rangle, \beta \in{ }_{M} \mathcal{X}_{M}^{\alpha}$. From (the proof of) [0, Prop. 3.1] we learn that

$$
v_{\beta}^{ \pm}=\left\{\begin{array}{cc}
d_{\beta} w / w_{+} & \beta \in{ }_{M} \mathcal{X}_{M}^{ \pm} \\
0 & \beta \notin_{M} \mathcal{X}_{M}^{ \pm}
\end{array} .\right.
$$

Consequently $\left\langle\vec{v}^{+}, \vec{v}^{-}\right\rangle=w_{0} w^{2} / w_{+}^{2}$. But we can also compute directly

$$
\left\langle\vec{v}^{+}, \vec{v}^{-}\right\rangle=\sum_{\lambda, \mu} \sum_{\beta \in_{M} \mathcal{X}_{M}^{0}} d_{\lambda}\left\langle\alpha_{\lambda}^{+}, \beta\right\rangle\left\langle\beta, \alpha_{\mu}^{-}\right\rangle d_{\mu}=\sum_{\lambda, \mu} d_{\lambda} Z_{\lambda, \mu} d_{\mu}=\zeta=\frac{w^{2}}{w_{\alpha}} .
$$

completing the proof.

Note that Proposition 3.1 in particular provides a new proof of the "generating property of $\alpha$-induction", i.e. ${ }_{M} \mathcal{X}_{M}^{\alpha}={ }_{M} \mathcal{X}_{M}$ if the braiding is non-degenerate, which was established in [6, Thm. 5.10].

Now recall that the chiral locality condition $\varepsilon^{+}(\theta, \theta) v^{2}=v^{2}$ expresses local commutativity ("locality") of the extended net, if $N \subset M$ arises from a net of subfactors [28.

Proposition 3.2 The following conditions are equivalent:

1. We have $Z_{\lambda, 0}=\langle\theta, \lambda\rangle$ for all $\lambda \in{ }_{N} \mathcal{X}_{N}$.

2. We have $Z_{0, \lambda}=\langle\theta, \lambda\rangle$ for all $\lambda \in{ }_{N} \mathcal{X}_{N}$.

3. Chiral locality holds: $\varepsilon^{+}(\theta, \theta) v^{2}=v^{2}$.

Proof. The implications $3 \Rightarrow 1$,2 follow from [3, Thm. 3.9]. We need to show 1,2 $\Rightarrow 3$. Recall $\langle\theta, \lambda\rangle=\langle\iota, \iota \lambda\rangle$. Moreover, by the extension property of $\alpha$-induction we have

$$
\operatorname{Hom}\left(\mathrm{id}, \alpha_{\lambda}^{ \pm}\right) \subset \operatorname{Hom}(\iota, \iota \lambda), \quad \lambda \in{ }_{N} \mathcal{X}_{N} .
$$

Hence, if $Z_{\lambda, 0}=\langle\theta, \lambda\rangle$ (respectively $Z_{0, \lambda}=\langle\theta, \lambda\rangle$ ) then $\operatorname{Hom}\left(\mathrm{id}, \alpha_{\lambda}^{ \pm}\right)=\operatorname{Hom}(\iota, \iota \lambda)$ for all $\lambda \in{ }_{N} \mathcal{X}_{N}$. Then consequently $\operatorname{Hom}\left(\mathrm{id}, \alpha_{\theta}^{ \pm}\right)=\operatorname{Hom}(\iota, \iota \theta)$. We clearly have $v \in \operatorname{Hom}(\iota, \iota \theta)$ and hence $v^{2}=\alpha_{\theta}^{ \pm}(v) v=\varepsilon^{ \pm}(\theta, \theta)^{*} v^{2}$, i.e. chiral locality holds.

Recall from [7, Prop. 3.4] that the coupling matrix arising from a braided subfactor with satisfied chiral locality condition is automatically of type I. Hence Proposition 3.2 states that chiral locality is equivalent to the canonical endomorphism being "fully visible" in the vacuum row (or column) of the coupling matrix. 


\section{Intermediate subfactors}

In this section we are searching for certain intermediate subfactors

$$
N \subset \tilde{M} \subset M
$$

of our subfactor $N \subset M$. It follows from [23, Sect. 3] that the set of such intermediate subfactors $\tilde{M}$ is in a bijective correspondence with systems of subspaces $\mathcal{K}_{\rho} \subset \mathcal{H}_{\rho}$, where $\mathcal{H}_{\rho}=\operatorname{Hom}(\iota, \iota \rho), \rho \in{ }_{N} \mathcal{X}_{N}$, and subject to conditions

(i.) $\mathcal{K}_{\rho}^{*} \subset N \mathcal{K}_{\bar{\rho}}$,

(ii.) $\mathcal{K}_{\rho} \mathcal{K}_{\sigma} \subset \sum_{\xi \prec \rho \sigma} N \mathcal{K}_{\xi}$,

where the sum in (ii.) runs over all $\xi \in{ }_{N} \mathcal{X}_{N}$ such that $N_{\rho, \sigma}^{\xi}>0$. The factor $\tilde{M}$ is then generated by $N$ and the $\mathcal{K}_{\rho}$ 's and is uniquely decomposed as

$$
\tilde{M}=\sum_{\rho} N \mathcal{K}_{\rho}
$$

The dual canonical endomorphism $\tilde{\theta}$ of $N \subset \tilde{M}$ decomposes as a sector as $[\tilde{\theta}]=$ $\bigoplus_{\rho} n_{\rho}[\rho]$, where $n_{\rho}=\operatorname{dim} \mathcal{K}_{\rho}$.

We now define the spaces

$$
\mathcal{K}_{\rho}^{ \pm}=\operatorname{Hom}\left(\mathrm{id}, \alpha_{\rho}^{ \pm}\right), \quad \rho \in{ }_{N} \mathcal{X}_{N} .
$$

Note that $\mathcal{K}_{\rho}^{ \pm} \subset \mathcal{H}_{\rho}=\operatorname{Hom}(\iota, \iota \rho)$, that $\operatorname{dim} \mathcal{K}_{\rho}^{+}=Z_{\rho, 0}$ and $\operatorname{dim} \mathcal{K}_{\rho}^{-}=Z_{0, \rho}$.

Lemma 4.1 We have

$$
\mathcal{K}_{\rho}^{ \pm}=\left\{z v: z \in \operatorname{Hom}(\theta, \rho), z \gamma(v)=z \varepsilon^{\mp}(\theta, \theta) \gamma(v)\right\}
$$

Proof. Let $x \in \mathcal{K}_{\rho}^{ \pm}$. Now $x$ is uniquely decomposed as $x=z v$ with $z \in N$. Clearly $z \in \operatorname{Hom}(\theta, \rho)$. Then $x \in \operatorname{Hom}\left(\mathrm{id}, \alpha_{\rho}^{ \pm}\right)$reads, using naturality (see e.g. [6, Eq. (8)]),

$$
z \gamma(v) v=z v^{2}=\alpha_{\rho}^{ \pm}(v) z v=\varepsilon^{\mp}(\theta, \rho) v z v=\varepsilon^{\mp}(\theta, \rho) \theta(z) \gamma(v) v=z \varepsilon^{\mp}(\theta, \theta) \gamma(v) v,
$$

hence is equivalent to $z \gamma(v)=z \varepsilon^{\mp}(\theta, \theta) \gamma(v)$.

We choose orthonormal bases of isometries $t\left({ }_{\rho, \sigma}^{\xi}\right)_{i} \in \operatorname{Hom}(\xi, \rho \sigma), i=1,2, \ldots, N_{\rho, \sigma}^{\xi}$ so that $\sum_{\xi} \sum_{i=1}^{N_{\rho, \sigma}^{\xi}} t\left({ }_{\rho, \sigma}^{\xi}\right)_{i} t\left({ }_{\rho, \sigma}^{\xi}\right)_{i}^{*}=\mathbf{1}$.

Lemma 4.2 We have

(i.) $\left(\mathcal{K}_{\rho}^{ \pm}\right)^{*}=r_{\rho}^{*} \mathcal{K}_{\bar{\rho}}^{ \pm}$, 
(ii.) $\mathcal{K}_{\rho}^{ \pm} \mathcal{K}_{\sigma}^{ \pm} \subset \sum_{\xi} \sum_{i=1}^{N_{\rho, \sigma}^{\xi}} t\left({ }_{\rho, \sigma}^{\xi}\right)_{i} \mathcal{K}_{\xi}^{ \pm}$.

Proof. Right Frobenius reciprocity [22] gives us an isomorphism Hom(id, $\left.\alpha \frac{ \pm}{\bar{\rho}}\right) \rightarrow$ $\operatorname{Hom}\left(\mathrm{id}, \alpha_{\rho}^{ \pm}\right), x \mapsto x^{*} r_{\rho}$. Thus any element $y \in \mathcal{K}_{\rho}^{ \pm}$can be written as $x^{*} r_{\rho}$ with some $x \in \mathcal{K}_{\bar{\rho}}^{ \pm}$, proving (i.). To prove (ii.), let $x_{\rho}=z_{\rho} v \in \mathcal{K}_{\rho}^{ \pm}$and $x_{\sigma}=z_{\sigma} v \in \mathcal{K}_{\sigma}^{ \pm}$be the decompositions according to Lemma 4.1. Then

$$
x_{\rho} x_{\sigma}=z_{\rho} v z_{\sigma} v=\sum_{\xi} \sum_{i=1}^{N_{\rho, \sigma}^{\xi}} t\left(\begin{array}{l}
\xi \\
\rho, \sigma
\end{array}\right)_{i} t\left(\xi_{\rho, \sigma}^{\xi}\right)_{i}^{*} z_{\rho} \theta\left(z_{\sigma}\right) \gamma(v) v .
$$

We first notice that $t\left({ }_{\rho, \sigma}^{\xi}\right)_{i}^{*} z_{\rho} \theta\left(z_{\sigma}\right) \gamma(v) \in \operatorname{Hom}(\theta, \xi)$. Next we check by use of the BFE and by $v^{2}=\gamma(v) v$ that

$$
\begin{aligned}
z_{\rho} \theta\left(z_{\sigma}\right) \gamma(v) \cdot \varepsilon^{\mp}(\theta, \theta) \gamma(v) & =z_{\rho} \theta\left(z_{\sigma}\right) \varepsilon^{\mp}\left(\theta, \theta^{2}\right) \theta(\gamma(v)) \gamma(v) \\
& =z_{\rho} \theta\left(z_{\sigma}\right) \theta\left(\varepsilon^{\mp}(\theta, \theta)\right) \varepsilon^{\mp}(\theta, \theta) \gamma(v)^{2} \\
& =\rho\left(z_{\sigma} \varepsilon^{\mp}(\theta, \theta)\right) z_{\rho} \varepsilon^{\mp}(\theta, \theta) \gamma(v)^{2} \\
& =z_{\rho} \theta\left(z_{\sigma}\right) \theta\left(\varepsilon^{\mp}(\theta, \theta)\right) \gamma(v)^{2} \\
& =z_{\rho} \theta\left(z_{\sigma} \varepsilon^{\mp}(\theta, \theta) \gamma(v)\right) \gamma(v)=z_{\rho} \theta\left(z_{\sigma}\right) \gamma(v) \cdot \gamma(v) .
\end{aligned}
$$

We conclude $x_{\rho} x_{\sigma}=\sum_{\xi} \sum_{i=1}^{N_{\rho, \sigma}^{\xi}} t\left({ }_{\rho, \sigma}^{\xi}\right)_{i} x_{\xi}^{i}$ with $x_{\xi}^{i}=t\left(\left(_{\rho, \sigma}^{\xi}\right)_{i}^{*} z_{\rho} \theta\left(z_{\sigma}\right) \gamma(v) v \in \mathcal{K}_{\xi}^{ \pm}\right.$.

Corollary 4.3 There are two (possibly identical) intermediate subfactors $N \subset M_{ \pm} \subset$ $M$ with $M_{ \pm}=\sum_{\rho} N \mathcal{K}_{\rho}^{ \pm}$.

Our next aim is to show that the subfactors $N \subset M_{ \pm}$obey the chiral locality condition. Let $\iota_{ \pm}: N \hookrightarrow M_{ \pm}$denote the injection maps, so that the (dual) canonical endomorphisms are given by $\gamma_{ \pm}=\iota_{ \pm} \bar{\iota}_{ \pm}$and $\theta_{ \pm}=\bar{\iota}_{ \pm} \iota_{ \pm}$. We now know that $\left[\theta_{+}\right]=$ $\bigoplus_{\rho} Z_{\rho, 0}[\rho]$ and $\left[\theta_{-}\right]=\bigoplus_{\rho} Z_{0, \rho}[\rho]$. Due to commutativity of $Y$ and $Z$, which yields in particular $\sum_{\rho} d_{\rho} Z_{\rho, 0}=\sum_{\rho} Z_{0, \rho} d_{\rho}$, we find $d_{\theta_{+}}=d_{\theta_{-}}$, i.e. the subfactors $N \subset M_{+}$ and $N \subset M_{-}$have the same Jones index. Moreover, we can apply $\alpha$-induction, i.e. we define morphisms in $\operatorname{End}\left(M_{\delta}\right)$ by

$$
\tilde{\alpha}_{\delta ; \lambda}^{ \pm}=\bar{\iota}_{\delta}^{-1} \circ \operatorname{Ad}\left(\varepsilon^{ \pm}\left(\lambda, \theta_{\delta}\right)\right) \circ \lambda \circ \bar{\iota}_{\delta},
$$

where the index $\delta$ is either $\delta=+$ or $\delta=-$. This will give rise to "parent" coupling matrices $Z^{\delta}$. Thanks to Prop. 3.2, it suffices to show $Z_{\lambda, 0}^{+}=Z_{\lambda, 0}$ and $Z_{0, \lambda}^{-}=Z_{0, \lambda}$, $\lambda \in{ }_{N} \mathcal{X}_{N}$, to prove that the chiral locality condition holds for $N \subset M_{+}$and $N \subset M_{-}$, respectively.

Lemma 4.4 We have $\tilde{\alpha}_{\delta ; \lambda}^{ \pm}\left(x_{\rho}\right)=\varepsilon^{ \pm}(\lambda, \rho)^{*} x_{\rho}$ for any $x_{\rho} \in \mathcal{K}_{\rho}^{\delta}$ and $\lambda, \rho \in{ }_{N} \mathcal{X}_{N}$. 
Proof. Let $x_{\rho}=z_{\rho} v$ be the decomposition according to Lemma 4.1. We first notice that

$$
\gamma_{\delta}\left(z_{\rho} v\right) \theta_{\delta}(n)=\gamma_{\delta}\left(z_{\rho} \theta(n) v\right)=\gamma_{\delta}\left(\rho(n) z_{\rho} v\right)=\theta_{\delta} \rho(n) \gamma_{\delta}\left(z_{\rho} v\right)
$$

for any $n \in N$, i.e. $\gamma_{\delta}\left(x_{\rho}\right) \in \operatorname{Hom}\left(\theta_{\delta}, \theta_{\delta} \rho\right)$. Therefore we can compute

$$
\begin{aligned}
\gamma_{\delta}\left(\tilde{\alpha}_{\delta ; \lambda}^{ \pm}\left(x_{\rho}\right)\right) & =\varepsilon^{ \pm}\left(\lambda, \theta_{\delta}\right) \lambda \gamma_{\delta}\left(x_{\rho}\right) \varepsilon^{\mp}\left(\theta_{\delta}, \lambda\right)=\varepsilon^{\mp}\left(\theta_{\delta}, \lambda\right)^{*} \varepsilon^{\mp}\left(\theta_{\delta} \rho, \lambda\right) \gamma_{\delta}\left(x_{\rho}\right) \\
& =\varepsilon^{\mp}\left(\theta_{\delta}, \lambda\right)^{*} \varepsilon^{\mp}\left(\theta_{\delta}, \lambda\right) \theta_{\delta}\left(\varepsilon^{\mp}(\rho, \lambda)\right) \gamma_{\delta}\left(x_{\rho}\right)=\gamma_{\delta}\left(\varepsilon^{ \pm}(\lambda, \rho)^{*} x_{\rho}\right)
\end{aligned}
$$

and application of $\gamma_{\delta}^{-1}$ yields the statement.

In the same manner we obtain of course also $\alpha_{\lambda}^{ \pm}\left(x_{\rho}\right)=\varepsilon^{ \pm}(\lambda, \rho)^{*} x_{\rho}$ for any $x_{\rho} \in$ $\operatorname{Hom}(\iota, \iota \rho)$. Therefore we obtain immediately

Corollary 4.5 We have $\left.\alpha_{\lambda}^{ \pm}\right|_{M_{\delta}}=\tilde{\alpha}_{\delta ; \lambda}^{ \pm}$.

Hence $x_{\lambda} x_{\rho}=\alpha_{\lambda}^{ \pm}\left(x_{\rho}\right) x_{\lambda}=\tilde{\alpha}_{\delta ; \lambda}^{ \pm}\left(x_{\rho}\right) x_{\lambda}$ whenever $x_{\lambda} \in \mathcal{K}_{\lambda}^{ \pm}$and $x_{\rho} \in \mathcal{K}_{\rho}^{\delta}$, and thus in particular $\mathcal{K}_{\lambda}^{ \pm} \subset \operatorname{Hom}\left(\mathrm{id}_{M_{ \pm}}, \tilde{\alpha}_{ \pm ; \lambda}^{ \pm}\right)$. (Warning: Note that the super- and subscripts, referring to the \pm -induction respectively to the choice of the algebra $M_{ \pm}$, now have to be the same because we have $\mathcal{K}_{\lambda}^{+} \subset M_{+}$and $\mathcal{K}_{\lambda}^{-} \subset M_{-}$, but in general not the other way round. Here and in the following, any formula containing such combined \pm -indices has to be read in such a way that we either take all the upper or all the lower signs.) On the other hand we have

$$
\left\langle\operatorname{id}_{M_{ \pm}}, \tilde{\alpha}_{ \pm ; \lambda}^{ \pm}\right\rangle \leq\left\langle\theta_{ \pm}, \lambda\right\rangle=\operatorname{dim} \mathcal{K}_{\lambda}^{ \pm}
$$

Therefore we arrive in particular at

Corollary 4.6 We have $\operatorname{Hom}\left(\mathrm{id}_{M_{ \pm}}, \tilde{\alpha}_{ \pm ; \lambda}^{ \pm}\right)=\mathcal{K}_{\lambda}^{ \pm}$for any $\lambda \in{ }_{N} \mathcal{X}_{N}$.

Corollary 4.6 tells us in particular that $Z_{\lambda, 0}^{+}=Z_{\lambda, 0}$ and $Z_{0, \lambda}^{-}=Z_{0, \lambda}$, so that both subfactors $N \subset M_{ \pm}$must satisfy the chiral locality condition. In turn they must be irreducible by the argument of [3, Cor. 3.6]. We summarize the discussion in the following

Theorem 4.7 There are two (possibly identical) intermediate subfactors $N \subset M_{ \pm} \subset$ $M$. The irreducible subfactors $N \subset M_{+}$and $N \subset M_{-}$have the same Jones index, they both satisfy the chiral locality condition and consequently give rise to type I coupling matrices $Z^{ \pm}$. The latter are related to the coupling matrix of the full subfactor $N \subset M$ through $Z_{\lambda, 0}^{+}=Z_{\lambda, 0}$ and $Z_{0, \lambda}^{-}=Z_{0, \lambda}$. 


\section{Chiral fusion rule automorphisms}

We now investigate the relations between the chiral systems ${ }_{M} \mathcal{X}_{M}^{+},{ }_{M} \mathcal{X}_{M}^{-}$and ${ }_{M_{+}} \mathcal{X}_{M_{+}}^{+}$, $M_{-} \mathcal{X}_{M_{-}}^{-}$, respectively. Note that by [6, Prop. 3.1] and Theorem 4.7, they all must have the same chiral global index $w_{+}$.

Lemma 5.1 We have

$$
\operatorname{Hom}\left(\alpha_{\lambda}^{ \pm}, \alpha_{\mu}^{ \pm}\right)=\operatorname{Hom}\left(\tilde{\alpha}_{ \pm ; \lambda}^{ \pm}, \tilde{\alpha}_{ \pm ; \mu}^{ \pm}\right),
$$

in particular $\operatorname{Hom}\left(\alpha_{\lambda}^{ \pm}, \alpha_{\mu}^{ \pm}\right) \subset M_{ \pm}$, for any $\lambda, \mu \in \Sigma\left({ }_{N} \mathcal{X}_{N}\right)$.

Proof. First let $\xi \in \Sigma\left({ }_{N} \mathcal{X}_{N}\right)$. Then there are orthonormal bases of isometries $s_{\nu, i} \in$ $\operatorname{Hom}(\nu, \xi), \nu \in{ }_{N} \mathcal{X}_{N}, i=1,2, \ldots,\langle\nu, \xi\rangle$, such that $\sum_{\nu, i} s_{\nu, i} s_{\nu, i}^{*}=\mathbf{1}$. We may write $x \in \operatorname{Hom}\left(\mathrm{id}, \alpha_{\xi}^{ \pm}\right)$as $\sum_{\nu, i} s_{\nu, i} s_{\nu, i}^{*} x$, and we notice $s_{\nu, i}^{*} x \in \operatorname{Hom}\left(\mathrm{id}, \alpha_{\nu}^{ \pm}\right)=\operatorname{Hom}\left(\mathrm{id}, \tilde{\alpha}_{ \pm ; \nu}^{ \pm}\right)$, thanks to Corollary 4.6, so that $x \in \operatorname{Hom}\left(\mathrm{id}, \tilde{\alpha}_{ \pm ; \xi}^{ \pm}\right)$. The same argument works vice versa. Now let $\lambda, \mu \in \Sigma\left({ }_{N} \mathcal{X}_{N}\right)$. Then we have Frobenius isomorphisms

$$
\operatorname{Hom}\left(\alpha_{\lambda}^{ \pm}, \alpha_{\mu}^{ \pm}\right) \longrightarrow \operatorname{Hom}\left(\mathrm{id}, \alpha_{\bar{\lambda} \mu}^{ \pm}\right)=\operatorname{Hom}\left(\mathrm{id}, \tilde{\alpha}_{ \pm ; \bar{\lambda} \mu}^{ \pm}\right) \longrightarrow \operatorname{Hom}\left(\tilde{\alpha}_{ \pm ; \lambda}^{ \pm}, \tilde{\alpha}_{ \pm ; \mu}^{ \pm}\right)
$$

which map

$$
t \mapsto s=\sqrt{d_{\lambda} / d_{\mu}} \alpha_{\bar{\lambda}}^{ \pm}(t) r_{\lambda}, \quad s \mapsto t^{\prime}=\sqrt{d_{\lambda} d_{\mu}} \bar{r}_{\lambda}^{*} \tilde{\alpha}_{ \pm ; \lambda}^{ \pm}(s) .
$$

As we have

$$
t^{\prime}=d_{\lambda} \bar{r}_{\lambda}^{*} \tilde{\alpha}_{ \pm ; \lambda}^{ \pm}\left(\alpha_{\bar{\lambda}}^{ \pm}(t) r_{\lambda}\right)=d_{\lambda} \bar{r}_{\lambda}^{*} \alpha_{\lambda \bar{\lambda}}^{ \pm}(t) \lambda\left(r_{\lambda}\right)=t
$$

it follows $\operatorname{Hom}\left(\alpha_{\lambda}^{ \pm}, \alpha_{\mu}^{ \pm}\right)=\operatorname{Hom}\left(\tilde{\alpha}_{ \pm ; \lambda}^{ \pm}, \tilde{\alpha}_{ \pm ; \mu}^{ \pm}\right) \subset M_{ \pm}$.

Lemma 5.2 Each $\beta_{ \pm} \in{ }_{M} \mathcal{X}_{M}^{ \pm}$is equivalent to an extension of some $\tilde{\beta}_{ \pm} \in{ }_{M_{ \pm}} \mathcal{X}_{M_{ \pm}}^{ \pm}$. This association gives rise to bijections $\vartheta_{ \pm}:{ }_{M} \mathcal{X}_{M}^{ \pm} \rightarrow{ }_{M_{ \pm}} \mathcal{X}_{M_{ \pm}}^{ \pm}$.

Proof. Assume $\beta \equiv \beta_{+} \in{ }_{M} \mathcal{X}_{M}^{+}$, i.e. there is a $\lambda \in{ }_{N} \mathcal{X}_{N}$ and an isometry $t \in$ $\operatorname{Hom}\left(\beta, \alpha_{\lambda}^{+}\right)$. Then $t t^{*}$ is a minimal projection in $\operatorname{Hom}\left(\tilde{\alpha}_{+; \lambda}^{+}, \tilde{\alpha}_{+; \lambda}^{+}\right)$by Lemma 5.1. Hence there is a $\tilde{\beta} \in{ }_{M_{+}} \mathcal{X}_{M_{+}}^{+}$and an isometry $\tilde{t} \in \operatorname{Hom}\left(\tilde{\beta}, \tilde{\alpha}_{+; \lambda}^{+}\right)$such that $\tilde{t} \tilde{t}^{*}=t t^{*}$. Thus putting $\beta^{\prime}(m)=\tilde{t}^{*} \alpha_{\lambda}^{+}(m) \tilde{t}$ for $m \in M$ gives an equivalent endomorphism, as $\beta=\operatorname{Ad}(u) \circ \beta^{\prime}$ with the unitary $u=t^{*} \tilde{t} \in M$, and we clearly have $\left.\beta^{\prime}\right|_{M_{+}}=\tilde{\beta}$, thanks to Corollary 4.5. It remains to show that, if $\tilde{\beta}_{1}, \tilde{\beta}_{2} \in{ }_{M_{+}} \mathcal{X}_{M_{+}}^{+}$correspond this way to different $\beta_{1}, \beta_{2} \in{ }_{M} \mathcal{X}_{M}^{+}$, then $\tilde{\beta}_{1}$ and $\tilde{\beta}_{1}$ are disjoint. Let $t_{j} \in \operatorname{Hom}\left(\beta_{j}, \alpha_{\lambda_{j}}^{+}\right)$ and $\tilde{t}_{j} \in \operatorname{Hom}\left(\tilde{\beta}_{j}, \tilde{\alpha}_{+; \lambda_{j}}^{+}\right)$be isometries as above, i.e. $t_{j} t_{j}^{*}=\tilde{t}_{j} \tilde{t}_{j}^{*}, j=1,2$. Assume for contradiction that there is a unitary $q \in \operatorname{Hom}\left(\tilde{\beta}_{1}, \tilde{\beta}_{2}\right)$. But then $\tilde{t}_{2} q \tilde{t}_{1}^{*} \in$ $\operatorname{Hom}\left(\tilde{\alpha}_{+; \lambda_{1}}^{+}, \tilde{\alpha}_{+; \lambda_{2}}^{+}\right)=\operatorname{Hom}\left(\alpha_{\lambda_{1}}^{+}, \alpha_{\lambda_{2}}^{+}\right)$, so that $t_{2}^{*} \tilde{t}_{2} q \tilde{t}_{1}^{*} t_{1}$ is a unitary in $\operatorname{Hom}\left(\beta_{1}, \beta_{2}\right)$, in contradiction to $\beta_{1}, \beta_{2}$ being different elements in ${ }_{M} \mathcal{X}_{M}^{ \pm}$. Hence the association $\beta \mapsto \tilde{\beta}$ defines a bijection $\vartheta_{+}:{ }_{M} \mathcal{X}_{M}^{+} \rightarrow{ }_{M_{+}} \mathcal{X}_{M_{+}}^{+}$. The proof is completed by exchanging "+" by "-" signs. 
Lemma 5.3 The bijections $\vartheta_{ \pm}:{ }_{M} \mathcal{X}_{M}^{ \pm} \rightarrow{ }_{M_{ \pm}} \mathcal{X}_{M_{ \pm}}^{ \pm}$preserve the chiral branching,

$$
\left\langle\beta, \alpha_{\lambda}^{ \pm}\right\rangle=\left\langle\vartheta_{ \pm}(\beta), \tilde{\alpha}_{ \pm ; \lambda}^{ \pm}\right\rangle, \quad \lambda \in{ }_{N} \mathcal{X}_{N}, \quad \beta \in{ }_{M} \mathcal{X}_{M}^{ \pm},
$$

and the chiral fusion rules

$$
\left\langle\beta_{3}, \beta_{1} \beta_{2}\right\rangle=\left\langle\vartheta_{ \pm}\left(\beta_{3}\right), \vartheta_{ \pm}\left(\beta_{1}\right) \vartheta_{ \pm}\left(\beta_{2}\right)\right\rangle, \quad \beta_{1}, \beta_{2}, \beta_{3} \in{ }_{M} \mathcal{X}_{M}^{ \pm},
$$

and the statistical dimensions.

Proof. We just consider the "+" case, the proof for "-" is analogous. By Lemma 5.2 we may and do assume for simplicity that now all $\beta \in{ }_{M} \mathcal{X}_{M}^{+}$are choosen such that $\left.\beta\right|_{M_{+}}=\vartheta_{+}(\beta)$. This already forces equality of statistical dimensions $d_{\beta}=$ $d_{\vartheta_{+}(\beta)}$. Moreover, we just have $\operatorname{Hom}\left(\beta, \alpha_{\lambda}^{+}\right)=\operatorname{Hom}\left(\vartheta_{+}(\beta), \tilde{\alpha}_{+; \lambda}^{+}\right)$, giving Eq. (13). Given isometries $t_{j} \in \operatorname{Hom}\left(\beta_{j}, \alpha_{\lambda_{j}}^{+}\right)=\operatorname{Hom}\left(\vartheta_{+}\left(\beta_{j}\right), \tilde{\alpha}_{+; \lambda_{j}}^{+}\right), j=1,2,3$, and also $y \in$ $\operatorname{Hom}\left(\beta_{3}, \beta_{1} \beta_{2}\right)$, then we find similarly

$$
t_{1} \alpha_{\lambda_{1}}^{+}\left(t_{2}\right) y t_{3}^{*} \in \operatorname{Hom}\left(\alpha_{\lambda_{3}}^{+}, \alpha_{\lambda_{1}}^{+} \alpha_{\lambda_{2}}^{+}\right)=\operatorname{Hom}\left(\tilde{\alpha}_{+; \lambda_{3}}^{+}, \tilde{\alpha}_{+; \lambda_{1}}^{+} \tilde{\alpha}_{+; \lambda_{2}}^{+}\right)
$$

by Lemma 5.1. Therefore, by using $\alpha_{\lambda_{1}}^{+}\left(t_{2}\right)=\tilde{\alpha}_{+; \lambda_{1}}^{+}\left(t_{2}\right)$ we finally find that $y \in$ $\operatorname{Hom}\left(\vartheta_{+}\left(\beta_{3}\right), \vartheta_{+}\left(\beta_{1}\right) \vartheta_{+}\left(\beta_{2}\right)\right)$. The same argument works vice versa, so that the intertwiner spaces $\operatorname{Hom}\left(\beta_{3}, \beta_{1} \beta_{2}\right)$ and $\operatorname{Hom}\left(\vartheta_{+}\left(\beta_{3}\right), \vartheta_{+}\left(\beta_{1}\right) \vartheta_{+}\left(\beta_{2}\right)\right)$ are equal.

Lemma 5.4 The bijections $\vartheta_{ \pm}$restrict to bijections ${ }_{M} \mathcal{X}_{M}^{0} \rightarrow{ }_{M_{ \pm}} \mathcal{X}_{M_{ \pm}}^{0}$ of the ambichiral subsystems.

Proof. Let $\tau \in{ }_{M} \mathcal{X}_{M}^{0}$, i.e. there are isometries $s \in \operatorname{Hom}\left(\tau, \alpha_{\lambda}^{+}\right)$and $t \in \operatorname{Hom}\left(\tau, \alpha_{\mu}^{-}\right)$ for some $\lambda, \mu \in{ }_{N} \mathcal{X}_{N}$. Put $q=t s^{*} \in \operatorname{Hom}\left(\alpha_{\lambda}^{+}, \alpha_{\mu}^{-}\right)$. Then $q \in \operatorname{Hom}(\iota \lambda, \iota \mu)$ and

$$
q \varepsilon^{+}(\lambda, \rho)^{*} x_{\rho}=\varepsilon^{-}(\mu, \rho)^{*} x_{\rho} q
$$

whenever $x_{\rho} \in \operatorname{Hom}(\iota, \iota \rho)$. Hence, using Eq. (9), we calculate the left-hand side as

$$
q \varepsilon^{+}(\lambda, \rho)^{*} x_{\rho}=q \varepsilon^{-}(\rho, \lambda) x_{\rho}=\varepsilon^{-}(\rho, \mu) \alpha_{\rho}^{-}(q) x_{\rho}=\varepsilon^{+}(\mu, \rho)^{*} \alpha_{\rho}^{-}(q) x_{\rho} .
$$

Now let us specialize to the case $x_{\rho} \in \mathcal{K}_{\rho}^{-}$. Then $x_{\rho} q=\alpha_{\rho}^{-}(q) x_{\rho}$, so that

$$
\varepsilon^{+}(\mu, \rho)^{*} x_{\rho} q=\varepsilon^{-}(\mu, \rho)^{*} x_{\rho} q .
$$

It follows $\tilde{\alpha}_{-; \mu}^{+}(m) q=\tilde{\alpha}_{-; \mu}^{-}(m) q$ for all $m \in M_{-}$. But note that $q q^{*}=t t^{*}$ which lies in $\operatorname{Hom}\left(\tilde{\alpha}_{-; \mu}^{-}, \tilde{\alpha}_{-; \mu}^{-}\right)$by Lemma 5.1. Hence $\tilde{\alpha}_{-; \mu}^{+}(m) t t^{*}=\tilde{\alpha}_{-; \mu}^{-}(m) t t^{*}$ for all $m \in M_{-}$. We can similarly derive that $\tilde{\alpha}_{+; \lambda}^{+}(m) s s^{*}=\tilde{\alpha}_{+; \lambda}^{-}(m) s s^{*}$ for all $m \in M_{+}$. There are isometries $\tilde{t} \in \operatorname{Hom}\left(\vartheta_{-}(\tau), \tilde{\alpha}_{-; \mu}^{-}\right)$and $\tilde{s} \in \operatorname{Hom}\left(\vartheta_{+}(\tau), \tilde{\alpha}_{+; \lambda}^{+}\right)$such that $\tilde{t} \tilde{t}^{*}=t t^{*}$ and $\tilde{s} \tilde{s}^{*}=s s^{*}$. But we now find

$$
\vartheta_{-}(\tau)(m)=\tilde{t}^{*} \tilde{\alpha}_{-; \mu}^{-}(m) \tilde{t}=\tilde{t}^{*} \tilde{\alpha}_{-; \mu}^{+}(m) \tilde{t}, \quad m \in M_{-},
$$


as well as

$$
\vartheta_{+}(\tau)(m)=\tilde{s}^{*} \tilde{\alpha}_{+; \lambda}^{+}(m) \tilde{s}=\tilde{s}^{*} \tilde{\alpha}_{+; \mu}^{-}(m) \tilde{s}, \quad m \in M_{+},
$$

i.e. $\vartheta_{ \pm}(\tau) \in{ }_{M_{ \pm}} \mathcal{X}_{M_{ \pm}}^{0}$. Thus $\vartheta_{ \pm} \operatorname{map}{ }_{M} \mathcal{X}_{M}^{0}$ into ${ }_{M_{ \pm}} \mathcal{X}_{M_{ \pm}}^{0}$. But it follows from Proposition 3.1 and Theorem 4.7 that the systems ${ }_{M} \mathcal{X}_{M}^{0}$ and ${ }_{M_{ \pm}} \mathcal{X}_{M_{ \pm}}^{0}$ all have the same ambichiral global index $w_{0}$. This proves the lemma.

We now can state the precise relation between the coupling matrix $Z$, arising from $N \subset M$ and given as in Eq. (6), and its type I parents $Z^{ \pm}$arising from $N \subset M_{ \pm}$.

Theorem 5.5 The entries of the type I coupling matrices $Z^{ \pm}$arising from $N \subset M_{ \pm}$ can be written as

$$
Z_{\lambda, \mu}^{ \pm}=\sum_{\tau \in_{M} \mathcal{X}_{M}^{0}} b_{\tau, \lambda}^{ \pm} b_{\tau, \mu}^{ \pm}
$$

with chiral branching coefficients

$$
b_{\tau, \lambda}^{ \pm}=\left\langle\tau, \alpha_{\lambda}^{ \pm}\right\rangle, \quad \tau \in{ }_{M} \mathcal{X}_{M}^{0}, \quad \lambda \in{ }_{N} \mathcal{X}_{N} .
$$

If the two intermediate subfactors of Corollary 4.J are identical, $M_{+}=M_{-}$, (so that the parent coupling matrices coincide, $Z^{+}=Z^{-}$) then the entries of the coupling matrix $Z$ arising from the full $N \subset M$ can be written as

$$
Z_{\lambda, \mu}=\sum_{\tau \in_{M} \mathcal{X}_{M}^{0}} b_{\tau, \lambda}^{+} b_{\omega(\tau), \mu}^{+} .
$$

Here the permutation $\omega=\vartheta_{+}^{-1} \circ \vartheta_{-}$of ${ }_{M} \mathcal{X}_{M}^{0}$, satisfying $\omega(0)=0$, realizes an automorphism of the ambichiral fusion rules.

Proof. Since the chiral locality condition holds for $N \subset M_{ \pm}$we have

$$
\left\langle\tilde{\tau}_{ \pm}, \tilde{\alpha}_{ \pm ; \mu}^{+}\right\rangle=\left\langle\bar{\iota}_{ \pm} \tilde{\tau}_{ \pm} \iota_{ \pm}, \mu\right\rangle=\left\langle\tilde{\tau}_{ \pm}, \tilde{\alpha}_{ \pm ; \mu}^{-}\right\rangle
$$

for $\tilde{\tau}_{ \pm} \in{ }_{M_{ \pm}} \mathcal{X}_{M_{ \pm}}^{0}$ and $\mu \in{ }_{N} \mathcal{X}_{N}$, thanks to [5, Prop. 3.3]. Therefore

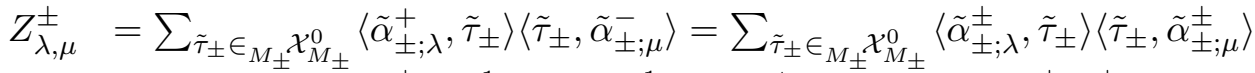

$$
\begin{aligned}
& =\sum_{\tilde{\tau}_{ \pm} \in_{M_{ \pm}} \mathcal{X}_{M_{ \pm}}^{0}}\left\langle\alpha_{\lambda}^{ \pm}, \vartheta_{ \pm}^{-1}\left(\tilde{\tau}_{ \pm}\right)\right\rangle\left\langle\vartheta_{ \pm}^{-1}\left(\tilde{\tau}_{ \pm}\right), \alpha_{\mu}^{ \pm}\right\rangle=\sum_{\tau \in \epsilon_{M}} \mathcal{X}_{M}^{0} b_{\tau, \lambda}^{ \pm} b_{\tau, \mu}^{ \pm}
\end{aligned}
$$

for $\lambda, \mu \in{ }_{N} \mathcal{X}_{N}$. Now if $M_{+}=M_{-}$then

$$
\begin{aligned}
Z_{\lambda, \mu} & =\sum_{\tau \epsilon_{M} \mathcal{X}_{M}^{0}}\left\langle\alpha_{\lambda}^{+}, \tau\right\rangle\left\langle\tau, \alpha_{\mu}^{-}\right\rangle=\sum_{\tau \epsilon_{M} \mathcal{X}_{M}^{0}} b_{\tau, \lambda}^{+}\left\langle\vartheta_{-}(\tau), \tilde{\alpha}_{-; \mu}^{-}\right\rangle \\
& =\sum_{\tau \epsilon_{M}} \mathcal{X}_{M}^{0} b_{\tau, \lambda}^{+}\left\langle\vartheta_{-}(\tau), \tilde{\alpha}_{+; \mu}^{+}\right\rangle=\sum_{\tau \epsilon_{M}} \mathcal{X}_{M}^{0} b_{\tau, \lambda}^{+}\left\langle\vartheta_{+}^{-1} \circ \vartheta_{-}(\tau), \alpha_{\mu}^{+}\right\rangle
\end{aligned}
$$

for $\lambda, \mu \in{ }_{N} \mathcal{X}_{N}$. As $M_{+}=M_{-}$, putting $\omega=\vartheta_{+}^{-1} \circ \vartheta_{-}$gives a well-defined permutation of ${ }_{M} \mathcal{X}_{M}^{0}$ and yields the desired formula $Z_{\lambda, \mu}=\sum_{\tau \in_{M}} \mathcal{X}_{M}^{0} b_{\tau, \lambda}^{+} b_{\omega(\tau), \mu}^{+}$. Due to Lemma 5.3, $\omega$ preserves the fusion rules and we also have $\omega(0)=0$ because always $\vartheta_{ \pm}\left(\mathrm{id}_{M}\right)=$ $\operatorname{id}_{M_{ \pm}}$. 
Note that even if $M_{+} \neq M_{-}, Z^{+} \neq Z^{-}$, the coupling matrix $Z$ is still governed by an isomorphism of (ambichiral) fusion rule algebras, in perfect agreement with [29]. Namely, if we use the system $\tilde{\tau}_{+} \in{ }_{M_{+}} \mathcal{X}_{M_{+}}^{0}$ for the summation in $Z$, then the general formula Eq. (6) can be written as

$$
Z_{\lambda, \mu}=\sum_{\tilde{\tau}_{+} \in \in_{M_{+}} \mathcal{X}_{M_{+}}^{0}}\left\langle\tilde{\alpha}_{+; \lambda}^{+}, \tilde{\tau}_{+}\right\rangle\left\langle\vartheta\left(\tilde{\tau}_{+}\right), \tilde{\alpha}_{-; \mu}^{-}\right\rangle=\sum_{\tilde{\tau}_{+} \in \in_{M_{+}} \mathcal{X}_{M_{+}}^{0}}\left\langle\lambda, \bar{\tau}_{+} \tilde{\tau}_{+} \iota_{+}\right\rangle\left\langle\tau_{-} \vartheta\left(\tilde{\tau}_{+}\right) \iota_{-}, \mu\right\rangle
$$

for $\lambda, \mu \in{ }_{N} \mathcal{X}_{N}$, by virtue of Lemma 5.3 and chiral locality, guaranteeing [5, Prop. 3.3]. Here $\vartheta$ is the bijection $\vartheta=\vartheta_{-} \circ \vartheta_{+}^{-1}:{ }_{M_{+}} \mathcal{X}_{M_{+}}^{0} \rightarrow_{M_{-}} \mathcal{X}_{M_{-}}^{0}$, yielding an isomorphism of the ambichiral fusion rules. This corresponds to the "extended" coupling matrix

$$
Z_{\tilde{\tau}_{+}, \tilde{\tau}_{-}}^{\mathrm{ext}}=\delta_{\tilde{\tau}_{-}, \vartheta\left(\tilde{\tau}_{+}\right)}, \quad \tilde{\tau}_{+} \in{ }_{M_{+}} \mathcal{X}_{M_{+}}^{0}, \quad \tilde{\tau}_{-} \in{ }_{M_{-}} \mathcal{X}_{M_{-}}^{0},
$$

which now has different left and right labels. (The reader may think of left and right extended characters $\chi_{\tilde{\tau}_{+}}^{\text {ext; } \pm}=\sum_{\lambda}\left\langle\lambda, \bar{t}_{ \pm} \tilde{\tau}_{ \pm} \iota_{ \pm}\right\rangle \chi_{\lambda}$ here.) This extended coupling matrix also appears in Eq. (20) below. Now only if $M_{+}=M_{-}$, so that ${ }_{M_{+}} \mathcal{X}_{M_{+}}^{0}={ }_{M_{-}} \mathcal{X}_{M_{-}}^{0}$, Eq. (18) can be reduced to $Z_{\tilde{\tau}_{+}, \tilde{\tau}_{+}^{\prime}}^{\text {ext }}=\delta_{\tilde{\tau}_{+}^{\prime}, \vartheta\left(\tilde{\tau}_{+}\right)}$which is nothing but the permutation $\omega$ up to relabeling by ${ }_{M} \mathcal{X}_{M}^{0}, \omega=\vartheta_{+}^{-1} \circ \vartheta \circ \vartheta_{+}$, which we used in Eq. (17) just for notational convenience.

Recall that there is a relative braiding between the morphisms in ${ }_{M} \mathcal{X}_{M}^{+}$and ${ }_{M} \mathcal{X}_{M}^{-}$ which restricts to a proper braiding on ${ }_{M} \mathcal{X}_{M}^{0}$, and for $\tau, \tau^{\prime} \in{ }_{M} \mathcal{X}_{M}^{0}$ these braiding operators are given by [5]

$$
\varepsilon_{\mathrm{r}}\left(\tau, \tau^{\prime}\right)=s^{*} \alpha_{\mu}^{-}(t)^{*} \varepsilon^{+}(\lambda, \mu) \alpha_{\lambda}^{+}(s) t
$$

whenever $t \in \operatorname{Hom}\left(\tau, \alpha_{\lambda}^{+}\right)$and $s \in \operatorname{Hom}\left(\tau^{\prime}, \alpha_{\mu}^{-}\right)$are isometries, $\lambda, \mu \in{ }_{N} \mathcal{X}_{N}$. We can extend this braiding from ${ }_{M} \mathcal{X}_{M}^{0}$ to $\Sigma\left({ }_{M} \mathcal{X}_{M}^{0}\right)$ as explained in [6, Sect. 2]. Now let $N^{\text {opp }}$ denote the opposite algebra of $N$ and let $j$ denote the natural anti-linear isomorphism. For $\lambda \in \operatorname{End}(N)$ we denote $\lambda^{\text {opp }}=j \circ \lambda \circ j$. We proceed analogously for $M_{-}^{\text {opp }}$, the opposite algebra of $M_{-}$.

Proposition 5.6 There exists a (type III) factor B such that we have irreducible inclusions

$$
N \otimes N^{\text {opp }} \subset M_{+} \otimes M_{-}^{\text {opp }} \subset B
$$

with the following properties:

1. The dual canonical endomorphism $\Theta_{\text {ext }}$ of the inclusion $M_{+} \otimes M_{-}^{\text {opp }} \subset B$ decomposes as

$$
\left[\Theta_{\mathrm{ext}}\right]=\bigoplus_{\tau \in \in_{M} \mathcal{X}_{M}^{0}}\left[\vartheta_{+}(\tau) \otimes \vartheta_{-}(\tau)^{\mathrm{opp}}\right]
$$

2. The dual canonical endomorphism $\Theta$ of the inclusion $N \otimes N^{\mathrm{opp}} \subset B$ decomposes as

$$
[\Theta]=\bigoplus_{\lambda, \mu \in \mathcal{X}_{N}} Z_{\lambda, \mu}\left[\lambda \otimes \mu^{\mathrm{opp}}\right]
$$


3. If $\tau_{ \pm}, \sigma_{ \pm} \in \operatorname{End}(M)$ are the extensions of $\tilde{\tau}_{ \pm}, \tilde{\sigma}_{ \pm} \in{ }_{M_{ \pm}} \mathcal{X}_{M_{ \pm}}^{0}$, respectively, according to Lemma 5.9, then

$$
y \sigma_{+}(x) \varepsilon_{\mathrm{r}}\left(\tilde{\tau}_{+}, \tilde{\sigma}_{+}\right)=\varepsilon_{\mathrm{r}}\left(\tilde{\tau}_{-}, \tilde{\sigma}_{-}\right) \tau_{-}(y) x
$$

holds whenever $x \in \operatorname{Hom}\left(\tau_{+}, \tau_{-}\right)$and $y \in \operatorname{Hom}\left(\sigma_{+}, \sigma_{-}\right)$.

Proof. Lemma 5.2 (together with Lemma 5.4) tells us that $\tilde{\tau}_{ \pm} \in{ }_{M_{ \pm}} \mathcal{X}_{M_{ \pm}}^{0}$ can be extended to $\tau_{ \pm} \in \operatorname{End}(M)$ such that $\tau_{+}$and $\tau_{-}$are equivalent to some morphisms in ${ }_{M} \mathcal{X}_{M}^{0}$, and we have $\left[\tau_{+}\right]=\left[\tau_{-}\right]$if and only if $\tilde{\tau}_{ \pm}=\vartheta_{ \pm}(\tau)$ for a $\tau \in{ }_{M} \mathcal{X}_{M}^{0}$. Using these extensions for subfactors $M_{ \pm} \subset M$ with systems ${ }_{M_{ \pm}} \mathcal{X}_{M_{ \pm}}^{0}$, then [35] determines a factor $B$ such that $M_{+} \otimes M_{-}^{\text {opp }} \subset B$ is an irreducible subfactor with its dual canonical endomorphism $\Theta_{\text {ext }}$ decomposing as

$$
\left[\Theta_{\mathrm{ext}}\right]=\bigoplus_{\tilde{\tau}_{+} \in \in_{M_{+}} \mathcal{X}_{M_{+}}^{0} \tilde{\tau}_{-} \in_{M_{-} \mathcal{X}_{M_{-}}^{0}}}\left\langle\tau_{+}, \tau_{-}\right\rangle\left[\tilde{\tau}_{+} \otimes \tilde{\tau}_{-}^{\mathrm{opp}}\right]=\bigoplus_{\tau \in \in_{M} \mathcal{X}_{M}^{0}}\left[\vartheta_{+}(\tau) \otimes \vartheta_{-}(\tau)^{\mathrm{opp}}\right]
$$

proving 1. Now note that the injection map for $N \otimes N^{\text {opp }} \hookrightarrow M_{+} \otimes M_{-}^{\text {opp }}$ is given by $\iota_{+} \otimes \iota_{-}^{\text {opp }}$. Therefore the dual canonical endomorphism for $N \otimes N^{\text {opp }} \subset B$ is obtained as $\Theta=\left(\bar{\iota}_{+} \otimes \bar{\iota}_{-}^{\text {opp }}\right) \circ \Theta^{\text {ext }} \circ\left(\iota_{+} \otimes \iota_{-}^{\text {opp }}\right)$ so that

$$
[\Theta]=\bigoplus_{\tau \in_{M} \mathcal{X}_{M}^{0}}\left[\left(\bar{\iota}_{+} \circ \vartheta_{+}(\tau) \circ \iota_{+}\right) \otimes\left(\bar{\iota}_{-} \circ \vartheta_{-}(\tau) \circ \iota_{-}\right)^{\mathrm{opp}}\right]
$$

Now

$$
\left[\bar{\iota}_{ \pm} \circ \vartheta_{ \pm}(\tau) \circ \iota_{ \pm}\right]=\bigoplus_{\lambda \in_{N} \mathcal{X}_{N}}\left\langle\bar{\iota}_{ \pm} \circ \vartheta_{ \pm}(\tau) \circ \iota_{ \pm}, \lambda\right\rangle[\lambda]
$$

and since the subfactors $N \subset M_{ \pm}$satisfy chiral locality one has

$$
\left\langle\bar{\iota}_{ \pm} \circ \vartheta_{ \pm}(\tau) \circ \iota_{ \pm}, \lambda\right\rangle=\left\langle\vartheta_{ \pm}(\tau), \tilde{\alpha}_{ \pm ; \lambda}^{ \pm}\right\rangle=\left\langle\tau, \alpha_{\lambda}^{ \pm}\right\rangle=b_{\tau, \lambda}^{ \pm}
$$

by virtue of [5, Prop. 3.3] and Lemma 5.3. Hence

$$
[\Theta]=\bigoplus_{\lambda, \mu \in N} \bigoplus_{\mathcal{X}_{N}} b_{\tau \in \in_{M} \mathcal{X}_{M}^{0}}^{+} b_{\tau, \mu}^{-}\left[\lambda \otimes \mu^{\mathrm{opp}}\right]=\bigoplus_{\lambda, \mu \in_{N} \mathcal{X}_{N}} Z_{\lambda, \mu}\left[\lambda \otimes \mu^{\mathrm{opp}}\right]
$$

proving 2. Finally, if $\tau_{ \pm}, \sigma_{ \pm} \in \operatorname{End}(M)$ denote the extensions of $\tilde{\tau}_{ \pm}, \tilde{\sigma}_{ \pm} \in{ }_{M_{ \pm}} \mathcal{X}_{M_{ \pm}}^{0}$, respectively, as in Lemma 5.2, then there are some $\lambda_{+}, \lambda_{-}, \mu_{+}, \mu_{-} \in{ }_{N} \mathcal{X}_{N}$ and isometries $\tilde{t}_{ \pm} \in \operatorname{Hom}\left(\tilde{\tau}_{ \pm}, \tilde{\alpha}_{ \pm ; \lambda_{ \pm}}^{ \pm}\right)$and $\tilde{s}_{ \pm} \in \operatorname{Hom}\left(\tilde{\sigma}_{ \pm}, \tilde{\alpha}_{ \pm ; \mu_{ \pm}}^{ \pm}\right)$so that $\tau_{ \pm}(m)=\tilde{t}_{ \pm}^{*} \alpha_{\lambda_{ \pm}}^{ \pm}(m) \tilde{t}_{ \pm}$ and $\sigma_{ \pm}(m)=\tilde{s}_{ \pm}^{*} \alpha_{\mu_{ \pm}}^{ \pm}(m) \tilde{s}_{ \pm}$for all $m \in M$. Note that also $\tilde{t}_{ \pm} \in \operatorname{Hom}\left(\tilde{\tau}_{ \pm}, \tilde{\alpha}_{ \pm ; \lambda_{ \pm}}^{\mp}\right)$ and $\tilde{s}_{ \pm} \in \operatorname{Hom}\left(\tilde{\sigma}_{ \pm}, \tilde{\alpha}_{ \pm ; \mu_{ \pm}}^{\mp}\right)$ because chiral locality holds for $N \subset M_{ \pm}$and then ambichiral morphisms are obtained from $\alpha^{+}$- and $\alpha^{-}$-induction by use of the same isometries $[5$, Sect. 3]. Hence we have

$$
\begin{aligned}
\varepsilon_{\mathrm{r}}\left(\tilde{\tau}_{ \pm}, \tilde{\sigma}_{ \pm}\right) & =\tilde{s}_{ \pm}^{*} \tilde{\alpha}_{ \pm ; \mu_{ \pm}}^{ \pm}\left(\tilde{t}_{ \pm}\right)^{*} \varepsilon^{+}\left(\lambda_{ \pm}, \mu_{ \pm}\right) \tilde{\alpha}_{ \pm ; \lambda_{ \pm}}^{ \pm}\left(\tilde{s}_{ \pm}\right) \tilde{t}_{ \pm} \\
& =\tilde{s}_{ \pm}^{*} \alpha_{\mu_{ \pm}}^{ \pm}\left(\tilde{t}_{ \pm}\right)^{*} \varepsilon^{+}\left(\lambda_{ \pm}, \mu_{ \pm}\right) \alpha_{\lambda_{ \pm}^{ \pm}}^{ \pm}\left(\tilde{s}_{ \pm}\right) \tilde{t}_{ \pm},
\end{aligned}
$$


where we also used Corollary 4.5. We now can compute

$$
\begin{aligned}
y \sigma_{+}(x) \varepsilon_{\mathrm{r}}\left(\tilde{\tau}_{+}, \tilde{\sigma}_{+}\right) & =y \sigma_{+}(x) \tilde{s}_{+}^{*} \alpha_{\mu_{+}}^{+}\left(\tilde{t}_{+}\right)^{*} \varepsilon^{+}\left(\lambda_{+}, \mu_{+}\right) \alpha_{\lambda_{+}}^{+}\left(\tilde{s}_{+}\right) \tilde{t}_{+} \\
& =y \sigma_{+}\left(x \tilde{t}_{+}^{*}\right) \tilde{s}_{+}^{*} \varepsilon^{+}\left(\lambda_{+}, \mu_{+}\right) \alpha_{\lambda_{+}}^{+}\left(\tilde{s}_{+}\right) \tilde{t}_{+} \\
& =\sigma_{-}\left(x \tilde{t}_{+}^{*}\right) \tilde{s}_{-}^{*} \tilde{s}_{-} y \tilde{s}_{+}^{*} \varepsilon^{+}\left(\lambda_{+}, \mu_{+}\right) \alpha_{\lambda_{+}}^{+}\left(\tilde{s}_{+}\right) \tilde{t}_{+} \\
& =\tilde{s}_{-}^{*} \alpha_{\mu_{-}}^{-}\left(\tilde{t}_{-}^{*} \tilde{t}_{-} x \tilde{t}_{+}^{*}\right) \varepsilon^{+}\left(\lambda_{+}, \mu_{-}\right) \alpha_{\lambda_{+}}^{+}\left(\tilde{s}_{-} y\right) \tilde{t}_{+} \\
& =\tilde{s}_{-}^{*} \alpha_{\mu_{-}}^{-}\left(\tilde{t}_{-}^{*}\right) \varepsilon^{+}\left(\lambda_{-}, \mu_{-}\right) \tilde{t}_{-} x \tilde{t}_{+}^{*} \alpha_{\lambda_{+}}^{+}\left(\tilde{s}_{-}\right) \tilde{t}_{+} \tau_{+}(y) \\
& =\tilde{s}_{-}^{*} \alpha_{\mu_{-}}^{-}\left(\tilde{t}_{-}^{*}\right) \varepsilon^{+}\left(\lambda_{-}, \mu_{-}\right) \alpha_{\lambda_{-}}^{-}\left(\tilde{s}_{-}\right) \tilde{t}_{-} x \tau_{+}(y)=\varepsilon_{\mathrm{r}}\left(\tilde{\tau}_{-}, \tilde{\sigma}_{-}\right) \tau_{-}(y) x,
\end{aligned}
$$

where we used Eq. (9) twice, proving 3.

The relevance of Proposition 5.6 is the following. Suppose that our factor $N$ is obtained as a local factor $N=N\left(I_{\circ}\right)$ of a quantum field theoretical net of factors $\{N(I)\}$ indexed by proper intervals $I \subset \mathbb{R}$ on the real line, and that the system ${ }_{N} \mathcal{X}_{N}$ is obtained as restrictions of DHR-morphisms (cf. [21]) to $N$. This is in fact the case in our RCFT examples arising from current algebras where the net is defined in terms of local loop groups in the vacuum representation. Taking two copies of such a net and placing the real axes on the light cone, then this defines a local net $\{A(\mathcal{O})\}$, indexed by double cones $\mathcal{O}$ on two-dimensional Minkowski space (cf. 34 for such constructions). Given a subfactor $N \subset M$, determining in turn two subfactors $N \subset M_{ \pm}$obeying chiral locality, will provide two local nets of subfactors $\left\{N(I) \subset M_{ \pm}(I)\right\}$ due to [28]. Arranging $M_{+}(I)$ and $M_{-}(J)$ on the two light cone axes defines a local net of subfactors $\left\{A(\mathcal{O}) \subset A_{\text {ext }}(\mathcal{O})\right\}$ in Minkowski space. The embedding $M_{+} \otimes M_{-}^{\text {opp }} \subset B$ gives rise to another net of subfactors $\left\{A_{\text {ext }}(\mathcal{O}) \subset B(\mathcal{O})\right\}$, and Eq. (22) ensures that the net $\{B(\mathcal{O})\}$ satisfies locality, due to Rehren's recent result [35]. As already shown in [35], there exist a local two-dimensional quantum field theory such that the coupling matrix $Z$ describes its restriction to the tensor products of its chiral building blocks $N(I)$, and this is here expressed in Eq. (21). Now Eq. (20) tells us that there are chiral extensions $N(I) \subset M_{+}(I)$ and $N(I) \subset M_{-}(I)$ for left and right chiral nets which are indeed maximal as the coupling matrix for $\left\{A_{\text {ext }}(\mathcal{O}) \subset B(\mathcal{O})\right\}$ is a bijection. This shows that the inclusions $N \subset M_{ \pm}$should in fact be regarded as the subfactor version of left- and right maximal extensions of the chiral algebra.

\section{Extended S- and T-matrices}

Using the braiding arising from the relative braiding one can define the statistics phase $\omega_{\tau}$ of $\tau \in{ }_{M} \mathcal{X}_{M}^{0}$ by $d_{\tau} \phi_{\tau}\left(\varepsilon_{\mathrm{r}}(\tau, \tau)\right)=\omega_{\tau} \mathbf{1}$.

Lemma 6.1 Let $\tau \in{ }_{M} \mathcal{X}_{M}^{0}$ such that $[\tau]$ is a subsector of $\left[\alpha_{\lambda}^{+}\right]$and $\left[\alpha_{\mu}^{-}\right]$for some $\lambda, \mu \in{ }_{N} \mathcal{X}_{N}$. Then we have $\omega_{\lambda}=\omega_{\tau}=\omega_{\mu}$. 
Proof. Let $t \in \operatorname{Hom}\left(\tau, \alpha_{\lambda}^{+}\right)$and $s \in \operatorname{Hom}\left(\tau, \alpha_{\mu}^{-}\right)$be isometries. Then

$$
\begin{aligned}
\omega_{\tau} \mathbf{1} & =d_{\tau} \phi_{\tau}\left(\varepsilon_{\mathrm{r}}(\tau, \tau)\right)=d_{\tau} \phi_{\tau}\left(s^{*} \alpha_{\mu}^{-}(t)^{*} \varepsilon^{+}(\lambda, \mu) \alpha_{\lambda}^{+}(s) t\right) \\
& =d_{\tau} \phi_{\tau}\left(\tau(t)^{*} s^{*} \varepsilon^{+}(\lambda, \mu) t \tau(s)\right)=d_{\tau} t^{*} \phi_{\tau}\left(s^{*} \varepsilon^{+}(\lambda, \mu) t\right) s \\
& =d_{\lambda} t^{*} \phi_{\alpha_{\lambda}^{+}}\left(t s^{*} \varepsilon^{+}(\lambda, \mu)\right) s=d_{\lambda} t^{*} \phi_{\alpha_{\lambda}^{+}}\left(\varepsilon^{+}(\lambda, \lambda) \alpha_{\lambda}^{+}\left(t s^{*}\right)\right) s \\
& =d_{\lambda} t^{*} \phi_{\alpha_{\lambda}^{+}}\left(\varepsilon^{+}(\lambda, \lambda)\right) t s^{*} s,
\end{aligned}
$$

where we used Eq. (7), Eq. (8), and since $t s^{*} \in \operatorname{Hom}(\iota \mu, \iota \lambda)$ we could also apply Eq. (9). Note that $\phi_{\alpha_{\lambda}^{+}}$can be given as $\phi_{\alpha_{\lambda}^{+}}(m)=r_{\lambda}^{*} \alpha_{\bar{\lambda}}^{+}(m) r_{\lambda}$ for all $m \in M$, so that in particular $\phi_{\alpha_{\lambda}^{+}}(n)=\phi_{\lambda}(n)$ for $n \in N$. Hence $\omega_{\tau}=\omega_{\lambda}$. We can compute similarly

$$
d_{\tau} t^{*} \phi_{\tau}\left(s^{*} \varepsilon^{+}(\lambda, \mu) t\right) s=d_{\mu} t^{*} \phi_{\alpha_{\mu}^{-}}\left(\varepsilon^{+}(\lambda, \mu) t s^{*}\right) s=d_{\mu} t^{*} \phi_{\alpha_{\mu}^{-}}\left(\alpha_{\mu}^{-}\left(t s^{*}\right) \varepsilon^{+}(\mu, \mu)\right) s,
$$

establishing $\omega_{\tau}=\omega_{\mu}$.

Note that with the expansion Eq. (6), Lemma 6.1 implies easily $\omega_{\lambda} Z_{\lambda, \mu}=Z_{\lambda, \mu} \omega_{\mu}$, i.e. it gives a new and simple proof of the commutativity of the matrices $\Omega$ and $Z$ which was first established in 6, Thm. 5.7].

Lemma 6.2 For $\beta \in{ }_{M} \mathcal{X}_{M}$ we have

$$
\sum_{\lambda \in \mathcal{X}_{N} \mathcal{X}_{N}} \omega_{\lambda} d_{\lambda}\left\langle\beta, \alpha_{\lambda}^{ \pm}\right\rangle=\left\{\begin{array}{cll}
\omega_{\tau} d_{\tau} w / w_{+} & : & \beta=\tau \in{ }_{M} \mathcal{X}_{M}^{0} \\
0 & : & \text { otherwise. }
\end{array}\right.
$$

Proof. All we need to show is that the left hand side of Eq. (23) vanishes whenever $\beta \notin{ }_{M} \mathcal{X}_{M}^{0}$ because we recall once more from (the proof of) [7, Prop. 3.1] that we have $d_{\tau} w / w_{+}=\sum_{\lambda} d_{\lambda} b_{\tau, \lambda}^{ \pm}$for any $\tau \in{ }_{M} \mathcal{X}_{M}^{0}$, and then the claim follows from Lemma 6.1. For this purpose we define vectors $\vec{u}^{ \pm}$with entries

$$
u_{\beta}^{ \pm}=\sum_{\lambda \in \in_{N} \mathcal{X}_{N}} \omega_{\lambda} d_{\lambda}\left\langle\beta, \alpha_{\lambda}^{ \pm}\right\rangle, \quad \beta \in{ }_{M} \mathcal{X}_{M} .
$$

We clearly have

$$
\begin{aligned}
\left\|\vec{u}^{+}\right\|^{2} & =\sum_{\beta} \sum_{\lambda, \nu} \omega_{\lambda} \omega_{\nu}^{-1} d_{\lambda} d_{\nu}\left\langle\alpha_{\nu}^{+}, \beta\right\rangle\left\langle\beta, \alpha_{\lambda}^{+}\right\rangle=\sum_{\lambda, \nu} \omega_{\lambda} \omega_{\nu}^{-1} d_{\lambda} d_{\nu}\left\langle\alpha_{\frac{\lambda}{\lambda}}^{+} \alpha_{\nu}^{+}, \mathrm{id}\right\rangle \\
& =\sum_{\lambda, \mu, \nu} \omega_{\lambda} \omega_{\nu}^{-1} d_{\lambda} d_{\nu} N_{\bar{\lambda}, \nu}^{\mu}\left\langle\alpha_{\mu}^{+}, \mathrm{id}\right\rangle=\sum_{\lambda, \mu, \nu} \omega_{\lambda} \omega_{\nu}^{-1} d_{\lambda} d_{\nu} N_{\lambda, \mu}^{\nu} \omega_{\mu} Z_{\mu, 0} \\
& =\sum_{\lambda, \mu} Y_{0, \lambda} Y_{\lambda, \mu} Z_{\mu, 0},
\end{aligned}
$$

where we used that $Z_{\mu, 0}=\omega_{\mu} Z_{\mu, 0}$ by Lemma 6.1. Similarly we obtain $\left\|\vec{u}^{-}\right\|^{2}=$ $\sum_{\lambda, \mu} Y_{0, \lambda} Y_{\lambda, \mu} Z_{0, \mu}$. On the other hand, we can compute the inner product

$$
\begin{aligned}
\left\langle\vec{u}^{+}, \vec{u}^{-}\right\rangle & =\sum_{\lambda, \mu} \sum_{\beta} \omega_{\lambda}^{-1} d_{\lambda} d_{\mu} \omega_{\mu}\left\langle\alpha_{\lambda}^{+}, \beta\right\rangle\left\langle\beta, \alpha_{\mu}^{-}\right\rangle=\sum_{\lambda, \mu} \omega_{\lambda}^{-1} d_{\lambda} d_{\mu} \omega_{\mu} Z_{\lambda, \mu} \\
& =\sum_{\lambda, \mu} d_{\lambda} Z_{\lambda, \mu} d_{\mu}=\sum_{\lambda, \mu} Y_{0, \lambda} Z_{\lambda, \mu} Y_{\mu, 0}=\sum_{\lambda, \mu} Y_{0, \lambda} Y_{\lambda, \mu} Z_{\mu, 0}=\left\|\vec{u}^{+}\right\|^{2},
\end{aligned}
$$


where we used the commutativity of $\Omega$ and $Y$ with $Z$ of [6, Thm. 5.7]. Commuting $Z$ in the fifth equality to the left rather than to the right gives $\left\langle\vec{u}^{+}, \vec{u}^{-}\right\rangle=\left\|\vec{u}^{-}\right\|^{2}$ Thus we conclude $\vec{u}^{+}=\vec{u}^{-}$. Since obviously $u_{\beta}^{ \pm}=0$ whenever $\beta \notin{ }_{M} \mathcal{X}_{M}^{ \pm}$this implies $u_{\beta}^{ \pm}=0$ whenever $\beta \notin{ }_{M} \mathcal{X}_{M}^{0}$. ${ }_{M} \mathcal{X}_{M}^{0}$.

Let $Y^{\text {ext }}$ and $\Omega^{\text {ext }}$ denote the Y- and $\Omega$-matrices associated to the braided system

Lemma 6.3 We have

$$
\frac{w}{w_{+}} \sum_{\tau^{\prime} \in_{M} \mathcal{X}_{M}^{0}} Y_{\tau, \tau^{\prime}}^{\text {ext }} b_{\tau^{\prime}, \mu}^{ \pm}=\sum_{\lambda \in \mathcal{X}_{N}} b_{\tau, \lambda}^{ \pm} Y_{\lambda, \mu}, \quad \sum_{\tau^{\prime} \in \in_{M} \mathcal{X}_{M}^{0}} \Omega_{\tau, \tau^{\prime}}^{\mathrm{ext}} b_{\tau^{\prime}, \mu}^{ \pm}=\sum_{\lambda \in \in_{N} \mathcal{X}_{N}} b_{\tau, \lambda}^{ \pm} \Omega_{\lambda, \mu}
$$

for all $\tau \in{ }_{M} \mathcal{X}_{M}^{0}$ and $\mu \in{ }_{N} \mathcal{X}_{N}$.

Proof. Note that the second relation in Eq. (24) is nothing but Lemma 6.1, as this is just $\omega_{\tau} b_{\tau, \lambda}^{ \pm}=b_{\tau, \lambda}^{ \pm} \omega_{\lambda}$. So we just need to verify the first relation in Eq. (24). We now compute

$$
\begin{aligned}
\sum_{\lambda} b_{\tau, \lambda}^{ \pm} Y_{\lambda, \mu} & =\sum_{\lambda, \rho} \frac{\omega_{\lambda} \omega_{\mu}}{\omega_{\rho}} N_{\lambda, \mu}^{\rho} d_{\rho} b_{\tau, \lambda}^{ \pm}=\sum_{\lambda, \rho} \frac{\omega_{\tau} \omega_{\mu}}{\omega_{\rho}} N_{\rho, \bar{\mu}}^{\lambda} d_{\rho}\left\langle\alpha_{\lambda}^{ \pm}, \tau\right\rangle \\
& =\sum_{\rho} \frac{\omega_{\tau} \omega_{\mu}}{\omega_{\rho}} d_{\rho}\left\langle\alpha_{\rho}^{ \pm}, \tau \alpha_{\mu}^{ \pm}\right\rangle=\sum_{\rho} \sum_{\beta} \frac{\omega_{\tau} \omega_{\mu}}{\omega_{\rho}} d_{\rho}\left\langle\alpha_{\rho}^{ \pm}, \beta\right\rangle\left\langle\beta, \tau \alpha_{\mu}^{ \pm}\right\rangle \\
& =\frac{w}{w_{+}} \sum_{\tau^{\prime \prime}} \frac{\omega_{\tau} \omega_{\mu}}{\omega_{\tau^{\prime \prime}}} d_{\tau^{\prime \prime}}\left\langle\tau^{\prime \prime}, \tau \alpha_{\mu}^{ \pm}\right\rangle=\frac{w}{w_{+}} \sum_{\tau^{\prime}, \tau^{\prime \prime}} \frac{\omega_{\tau} \omega_{\mu}}{\omega_{\tau^{\prime \prime}}} N_{\bar{\tau}, \tau^{\prime \prime}}^{\tau^{\prime}} d_{\tau^{\prime \prime}}\left\langle\tau^{\prime}, \alpha_{\mu}^{ \pm}\right\rangle \\
& =\frac{w}{w_{+}} \sum_{\tau^{\prime}, \tau^{\prime \prime}} \frac{\omega_{\tau} \omega_{\tau^{\prime}}}{\omega_{\tau^{\prime \prime}}} N_{\tau, \tau^{\prime}}^{\tau^{\prime \prime}} d_{\tau^{\prime \prime}} b_{\tau^{\prime}, \mu}^{ \pm}=\frac{w}{w_{+}} \sum_{\tau^{\prime}} Y_{\tau, \tau^{\prime}}^{\text {ext }} b_{\tau^{\prime}, \mu}^{ \pm}
\end{aligned}
$$

where we used (the complex conjugate of) Lemma 6.2 in the fifth equality.

Recall from Section $⿴$ the complex number $z=\sum_{\lambda \in_{N} \mathcal{X}_{N}} d_{\lambda}^{2} \omega_{\lambda}$. Analogously we put $z_{0}=\sum_{\tau \in \in_{M}} \mathcal{X}_{M}^{0} d_{\tau}^{2} \omega_{\tau}$.

Lemma 6.4 We have $z_{0}=\frac{w_{+}}{w} z$.

Proof. Using Lemma 6.2 we can compute

$$
z_{0}=\sum_{\tau \in \in_{M} \mathcal{X}_{M}^{0}} \omega_{\tau} d_{\tau}^{2}=\frac{w_{+}}{w} \sum_{\beta \in \in_{M} \mathcal{X}_{M}} \sum_{\lambda \in \in_{N} \mathcal{X}_{N}} \omega_{\lambda} d_{\lambda}\left\langle\beta, \alpha_{\lambda}^{ \pm}\right\rangle d_{\beta}=\frac{w_{+}}{w} \sum_{\lambda \in \mathcal{X}_{N} \mathcal{X}_{N}} \omega_{\lambda} d_{\lambda}^{2}=\frac{w_{+}}{w} z,
$$

where we used $\sum_{\beta \in{ }_{M} \mathcal{X}_{M}}\left\langle\beta, \alpha_{\lambda}^{ \pm}\right\rangle d_{\beta}=d_{\lambda}$. 
Assume that $z \neq 0$ so that the central charge can be defined. Since $w / w_{+}$is a real number, Lemma 6.4 tells us that the central charges $c$ and $c_{\text {ext }}$ of the braided systems ${ }_{N} \mathcal{X}_{N}$ and ${ }_{M} \mathcal{X}_{M}^{0}$, respectively, which are defined modulo 8, coincide. As a corollary of Lemma 6.3, the intertwining properties of chiral branching coefficients between original and extended S- and T-matrices are therefore clarified in the following

Theorem 6.5 Provided $z \neq 0$ one has

$$
\sum_{\tau^{\prime} \in_{M} \mathcal{X}_{M}^{0}} S_{\tau, \tau^{\prime}}^{\text {ext }} b_{\tau^{\prime}, \mu}^{ \pm}=\sum_{\lambda \in_{N} \mathcal{X}_{N}} b_{\tau, \lambda}^{ \pm} S_{\lambda, \mu}, \quad \sum_{\tau^{\prime} \in_{M} \mathcal{X}_{M}^{0}} T_{\tau, \tau^{\prime}}^{\text {ext }} b_{\tau^{\prime}, \mu}^{ \pm}=\sum_{\lambda \in_{N} \mathcal{X}_{N}} b_{\tau, \lambda}^{ \pm} T_{\lambda, \mu}
$$

for all $\tau \in{ }_{M} \mathcal{X}_{M}^{0}$ and $\mu \in{ }_{N} \mathcal{X}_{N}$.

We would like to remind the reader that, if the braiding on ${ }_{N} \mathcal{X}_{N}$ is non-degenerate, then so is the ambichiral braiding [0, Thm. 4.2]. In other words, whenever the original $\mathrm{S}$ - and T-matrices are modular then so are the extended S- and T-matrices.

Now let us return to our situation $N \subset M_{ \pm} \subset M$ and apply the above results also to the subfactors $N \subset M_{ \pm}$. Let $Y^{\text {ext; } \pm}$ and $\Omega^{\text {ext; } \pm}$ the $Y$ - and $\Omega$-matrices associated to the braided systems ${ }_{M_{+}} \mathcal{X}_{M_{ \pm}}^{0}$. Recalling now $Z_{\lambda, 0}^{+}=Z_{\lambda, 0}$ and $Z_{0, \lambda}^{-}=Z_{0, \lambda}$, we obtain from Lemmata 5.3, 5.4, 6.1 and 6.4 the following

Theorem 6.6 The matrices $Y^{\mathrm{ext}}, \Omega^{\mathrm{ext}}$, and $Y^{\mathrm{ext} ; \pm}, \Omega^{\mathrm{ext} ; \pm}$ coincide subject to the bijections $\vartheta_{ \pm}$. If $z \neq 0$, then so do the corresponding $S$ - and T-matrices which are then well-defined. In formulae,

$$
S_{\tau, \tau^{\prime}}^{\text {ext }}=S_{\vartheta_{ \pm}(\tau), \vartheta_{ \pm}\left(\tau^{\prime}\right)}^{\text {ext; }}, \quad T_{\tau, \tau^{\prime}}^{\text {ext }}=T_{\vartheta_{ \pm}(\tau), \vartheta_{ \pm}\left(\tau^{\prime}\right)}^{\text {ext; }},
$$

for all $\tau, \tau^{\prime} \in{ }_{M} \mathcal{X}_{M}^{0}$.

We remark that in the case that $M_{+}=M_{-}$one obtains by use of the properties of the relative braiding operators [5, Lemma 3.11] and from Corollary 4.5, that the ambichiral braiding operators are the same for ${ }_{M} \mathcal{X}_{M}^{0}$ and ${ }_{M_{ \pm}} \mathcal{X}_{M_{ \pm}}^{0}$, subject to the bijections $\vartheta_{ \pm}$, so that Theorem 6.6 is trivial in this case.

\section{Heterotic examples}

We consider the $S O(n)$ current algebra models at level 1 , and where $n$ is a multiple of $16, n=16 \ell, \ell=1,2,3, \ldots$. These theories have four sectors, the basic (0), vector (v), spinor (s) and conjugate spinor (c) module, corresponding to highest weights $0, \Lambda_{(1)}$, $\Lambda_{(r-1)}$ and $\Lambda_{(r)}$, respectively; here $r=n / 2=8 \ell$ is the rank of $S O(n)$. The conformal dimensions are given as $h_{0}=0, h_{\mathrm{v}}=1 / 2, h_{\mathrm{s}}=h_{\mathrm{c}}=\ell$, and the sectors obey $\mathbb{Z}_{2} \times \mathbb{Z}_{2}$ fusion rules. The Kac-Peterson matrices are given explicitly as

$$
S=\frac{1}{2}\left(\begin{array}{rrrr}
1 & 1 & 1 & 1 \\
1 & 1 & -1 & -1 \\
1 & -1 & 1 & -1 \\
1 & -1 & -1 & 1
\end{array}\right), \quad T=\mathrm{e}^{-2 \pi \mathrm{i} \ell / 3}\left(\begin{array}{rrrr}
1 & 0 & 0 & 0 \\
0 & -1 & 0 & 0 \\
0 & 0 & 1 & 0 \\
0 & 0 & 0 & 1
\end{array}\right)
$$


It is easy to check that there are exactly six modular invariants, $Z=\mathbf{1}, W, X_{\mathrm{s}}, X_{\mathrm{c}}$, $Q,{ }^{\mathrm{t}} Q$. Here

$$
W=\left(\begin{array}{cccc}
1 & 0 & 0 & 0 \\
0 & 1 & 0 & 0 \\
0 & 0 & 0 & 1 \\
0 & 0 & 1 & 0
\end{array}\right), \quad X_{\mathrm{s}}=\left(\begin{array}{cccc}
1 & 0 & 1 & 0 \\
0 & 0 & 0 & 0 \\
1 & 0 & 1 & 0 \\
0 & 0 & 0 & 0
\end{array}\right), \quad Q=\left(\begin{array}{cccc}
1 & 0 & 0 & 1 \\
0 & 0 & 0 & 0 \\
1 & 0 & 0 & 1 \\
0 & 0 & 0 & 0
\end{array}\right),
$$

and $X_{\mathrm{c}}=W X_{\mathrm{s}} W$. (Note that $Q=X_{\mathrm{s}} W$ and ${ }^{\mathrm{t}} Q=W X_{\mathrm{s}}$.) The matrix $Q$ and its transpose ${ }^{\mathrm{t}} Q$ are two examples for modular invariants with non-symmetric vacuum coupling. Such "heterotic" invariants seem to be extremely rare and have not enjoyed particular attention in the literature, perhaps because they were erroneously dismissed as being spurious in the sense that they would not correspond to a physical partition function. Examples for truly spurious modular invariants were given in [37, 39, 18] and found to be "coincidental" linear combinations of proper physical invariants. Note that although there is a linear dependence here, namely

$$
\mathbf{1}-W-X_{\mathrm{s}}-X_{\mathrm{c}}+Q+{ }^{\mathrm{t}} Q=0,
$$

we cannot express $Q$ ( or $^{\mathrm{t}} Q$ ) alone as a linear combination of the four symmetric invariants. This may serve as a first indication that $Q$ and ${ }^{\mathrm{t}} Q$ are not spurious. We will now demonstrate that they can be realized from subfactors.

The $\mathbb{Z}_{2} \times \mathbb{Z}_{2}$ fusion rules for these models were proven in the DHR framework in [2], and together with the conformal spin and statistics theorem [16, 15, 20] we conclude that there is a net of type III factors on $S^{1}$ with a system $\left\{\mathrm{id}, \rho_{\mathrm{v}}, \rho_{\mathrm{s}}, \rho_{\mathrm{c}}\right\}$ of localized and transportable, hence braided endomorphisms, such that the statistics S- and T-matrices are given by Eq. (27). Because the statistics phases are given as $\omega_{\mathrm{v}}=-1$ and $\omega_{\mathrm{s}}=\omega_{\mathrm{c}}=1$, we can assume that the morphisms in the system obey the $\mathbb{Z}_{2} \times \mathbb{Z}_{2}$ fusion rules even by individual multiplication,

$$
\rho_{\mathrm{v}}^{2}=\rho_{\mathrm{s}}^{2}=\rho_{\mathrm{c}}^{2}=\mathrm{id}, \quad \rho_{\mathrm{v}} \rho_{\mathrm{s}}=\rho_{\mathrm{s}} \rho_{\mathrm{v}}=\rho_{\mathrm{c}},
$$

thanks to [33, Lemma 4.4]. This is enough to proceed with the DHR construction of the field net [13], as already carried out similarly for simple current extensions with cyclic groups in 4 . 5]. In fact, all we need to do here is to pick a single local factor $N=N(I)$ such that the interval $I \subset S^{1}$ contains the localization region of the morphisms, and then we construct the cross product subfactor $N \subset N \rtimes\left(\mathbb{Z}_{2} \times \mathbb{Z}_{2}\right)$. Then the corresponding dual canonical endomorphism $\theta$ decomposes as a sector as

$$
[\theta]=[\mathrm{id}] \oplus\left[\rho_{\mathrm{v}}\right] \oplus\left[\rho_{\mathrm{s}}\right] \oplus\left[\rho_{\mathrm{c}}\right]
$$

Checking $\langle\iota \lambda, \iota \mu\rangle=\langle\theta \lambda, \mu\rangle=1$ for $\lambda, \mu=\mathrm{id}, \rho_{\mathrm{v}}, \rho_{\mathrm{s}}, \rho_{\mathrm{c}}$, we find that there is only a single $M-N$ sector, namely [ $\iota]$. By [6, Cor. 6.13] we conclude that the modular invariant coupling matrix $Z$ arising from this subfactor must fulfill $\operatorname{tr} Z=1$. This leaves only the possibility that $Z$ is $Q$ or ${ }^{\mathrm{t}} Q$. We may and do assume that $Z=Q$, otherwise we exchange braiding and opposite braiding. It is easy to determine the 
intermediate subfactors $N \subset M_{ \pm} \subset M$. Namely, we have $M_{+}=N \rtimes_{\rho_{\mathrm{s}}} \mathbb{Z}_{2}$ and $M_{-}=N \rtimes_{\rho_{\mathrm{c}}} \mathbb{Z}_{2}$ with dual canonical endomorphism sectors $\left[\theta_{+}\right]=[\mathrm{id}] \oplus\left[\rho_{\mathrm{s}}\right]$ and $\left[\theta_{-}\right]=[\mathrm{id}] \oplus\left[\rho_{\mathrm{c}}\right]$, respectively. That both extensions are local can also be checked from $\omega_{\mathrm{s}}=\omega_{\mathrm{c}}=1$. We therefore find $Z^{+}=X_{\mathrm{s}}$ and $Z^{-}=X_{\mathrm{c}}$. Finally, the permutation invariant $W$ is obtained from the non-local extension $M_{\mathrm{v}}=N \rtimes_{\rho_{\mathrm{v}}} \mathbb{Z}_{2}$.

\section{Conclusions}

We studied the structure of coupling matrices $Z$ arising from braided subfactors $N \subset M$ through intermediate subfactors $N \subset M_{ \pm} \subset M$ which in turn determine type I "parent" coupling matrices $Z^{ \pm}$. We demonstrated that the inclusions $N \subset M_{+}$ and $N \subset M_{-}$should be recognized as the subfactor version of left and right maximal chiral algebra extensions. The main application we have in mind is RCFT where the S- and T-matrices arising from the braiding are modular. For current algebra models based on Lie groups $S U(n)$ or others, the coupling matrices from subfactors are then modular invariants for their Kac-Peterson matrices. Most but not all of the known modular invariant coupling matrices of such models are either type I, Eq. (3), or type II, Eq. (4), and the type II invariants have a unique type I parent. For example, the parents of the $S U(2)$ type II modular invariants $\mathrm{D}_{2 \ell+1}(\ell=2,3, \ldots)$ and $\mathrm{E}_{7}$ are $\mathrm{A}_{4 \ell-1}$ and $\mathrm{D}_{10}$, respectively. For such invariants the extended left and right chiral algebras are the same. (In the $\mathrm{D}_{\text {odd }}$ examples the extended algebras are the original, identical left and right current algebras.) In fact, the $\mathrm{E}_{7}$ modular invariant has been constructed from a subfactor with $[\theta]=\left[\lambda_{0}\right] \oplus\left[\lambda_{8}\right] \oplus\left[\lambda_{16}\right]$ in [0], and here we obtain $M_{+}=M_{-}$with $\left[\theta_{ \pm}\right]=\left[\lambda_{0}\right] \oplus\left[\lambda_{16}\right]$ which produces the simple current extension $\mathrm{D}_{10}$ invariant. For the cases $\mathrm{D}_{\text {even }}, \mathrm{E}_{6}$ and $\mathrm{E}_{8}$ treated in [⿴囗十 , 5] where $N \subset M$ is subject to chiral locality from the beginning, we clearly find $M=M_{+}=M_{-}$which indeed are the local factors of the local chiral extensions considered e.g. in [36]. (In fact all invariants obtained from subfactors obeying chiral locality are clearly their own parents due to Proposition 3.2.) We showed that $Z$ is in fact type II, Eq. (田), whenever the extensions coincide, $M_{+}=M_{-}$.

It is interesting that all our results could be derived without assuming the nondegeneracy of the braiding, i.e. all our statements are true even if the modular group is not around. We similarly derived in [7] without such condition that trivial vacuum coupling, $Z_{\lambda, 0}=\delta_{\lambda, 0}$, is equivalent to $Z$ being a fusion rule automorphism (and to $Z_{0, \lambda}=\delta_{\lambda, 0}$ ), thus recovering a result previously encountered in RCFT [12, 29]. In this paper we started with a braided subfactor producing some coupling matrix with possibly non-trivial vacuum coupling, and our results show that the "extended" cou-

pling matrix, Eq. (18), is a bijection ${ }_{M_{+}} \mathcal{X}_{M_{+}}^{0} \rightarrow{ }_{M_{-}} \mathcal{X}_{M_{-}}^{0}$ which yields an isomorphism of the fusion rules of the ambichiral systems. Moreover, the corresponding extended S- and T-matrices coincide subject to this isomorphism (Theorem 6.6). In the (modular) RCFT case, they are recognized as the S- and T-matrices of the extended left and right chiral algebra, and therefore Theorem 6.6 provides in particular a subfactor version of [29, Eq. (4.5)]. But note that the derivations of the fusion rule automor- 
phism in [12, 29] in turn rely on the Verlinde formula [38] whereas our derivation holds even if the braiding is degenerate, i.e. even if the Verlinde formula does not hold. Our result comes in the same spirit as [34] where the embedding of left and right chiral observables in a $2 \mathrm{D}$ conformal quantum field theory is analyzed and the corresponding coupling matrix is shown to describe an automorphism of fusion rules if and only if the chiral observables are maximal. Namely, the result of [34] is derived under very general assumptions in the framework of local quantum physics, and it is in particular entirely independent of the $S L(2 ; \mathbb{Z})$ machinery heavily exploited in [12, 29].

Note that "almost all" known modular invariants satisfy $Z_{\lambda, 0}=Z_{0, \lambda}$. This means $\left[\theta_{+}\right]=\left[\theta_{-}\right]$, and this comes close to $M_{+}=M_{-}$. In particular, if $\langle\theta, \lambda\rangle \leq 1$, then this forces $\mathcal{K}_{\lambda}^{+}=\mathcal{K}_{\lambda}^{-}$so that we necessarily have $M_{+}=M_{-}$. Similarly a total degenerate braiding gives rise to $M_{+}=M_{-}$. Nevertheless our example in Section 7 has shown that $M_{+} \neq M_{-}$is possible, an that even $Z^{+} \neq Z^{-}$can occur. The significance of different left and right chiral extensions reflected in the possibility $M_{+} \neq M_{-}$and even in different parent coupling matrices may come a little surprising. For example, the related "heterotic" extensions of current algebra models are not particularly well studied objects. One reason may be that the most popular models, those based on $S U(n)$ current algebras, only seem to have modular invariants with identical parents - it is in fact likely that all $S U(n)$ invariants are entirely symmetric. [f But can it happen that $Z^{+}=Z^{-}$but $M_{+} \neq M_{-}$? We do not know an example but we neither see a reason why this should not be possible. For instance, if there is $\langle\lambda, \theta\rangle \geq 2$ for some $\lambda$ then it may happen that $\mathcal{K}_{\lambda}^{+} \neq \mathcal{K}_{\lambda}^{-}$though these spaces may still have the same dimension, $Z_{\lambda, 0}=Z_{0, \lambda}$. In other words, it is conceivable that certain modular invariants look like being type I or type II though they really come from heterotic extensions.

Let us finally mention that the exotic modular invariants which are argued not to correspond to any RCFT in [37, 39, 18], will not be produced from subfactors by the machinery of [6, 7]. Note that the standard argument showing that a modular invariant $Z$ does not give a partition function of a RCFT is to disprove the existence of an extended S-matrix. However, from braided subfactors there always arises a matrix $S^{\text {ext }}$ with all the required properties. And in fact, Rehren's recent result [35] (and in turn our Proposition 5.6) shows generally that all coupling matrices which arise from an embedding of some local algebra of a chiral RCFT describe the restriction of a 2D RCFT to its chiral building blocks. This implies for example that the heterotic modular invariants $Q$ and ${ }^{\mathrm{t}} Q$ discussed in Section 7 are in fact coupling matrices of a RCFT whose chiral algebras are different maximal extensions of the $S O(n)_{1}$ current algebra.

Acknowledgement. We would like to thank especially T. Gannon for drawing our attention to the non-symmetric $S O(16 \ell)_{1}$ modular invariants as well as K.-H. Rehren for pointing out the benefit of proving Proposition 5.6, and we are indebted to J. Fuchs, T. Gannon, and K.-H. Rehren for helpful e-mail correspondences. We gratefully acknowledge financial support of the EU TMR Network in Non-Commutative Geometry.

\footnotetext{
${ }^{2}$ This was pointed out to us by Terry Gannon.
} 


\section{References}

[1] Behrend, R.E., Pearce, P.A., Petkova, V.B., Zuber, J.-B.: Boundary conditions in rational conformal field theories. Preprint, hep-th/9908036

[2] Böckenhauer, J.: An algebraic formulation of level one Wess-Zumino-Witten models. Rev. Math. Phys. 8, 925-947 (1996)

[3] Böckenhauer, J., Evans, D.E.: Modular invariants, graphs and $\alpha$-induction for nets of subfactors. I. Commun. Math. Phys. 197, 361-386 (1998)

[4] Böckenhauer, J., Evans, D.E.: Modular invariants, graphs and $\alpha$-induction for nets of subfactors. II. Commun. Math. Phys. 200, 57-103 (1999)

[5] Böckenhauer, J., Evans, D.E.: Modular invariants, graphs and $\alpha$-induction for nets of subfactors. III. Commun. Math. Phys. 205, 183-228 (1999)

[6] Böckenhauer, J., Evans, D.E., Kawahigashi, Y.: On $\alpha$-induction, chiral generators and modular invariants for subfactors. Commun. Math. Phys. 208, 429-487 (1999)

[7] Böckenhauer, J., Evans, D.E., Kawahigashi, Y.: Chiral structure of modular invariants for subfactors. Preprint, math.OA/9907149

[8] Cappelli, A., Itzykson, C., Zuber, J.-B.: The $A-D-E$ classification of minimal and $A_{1}^{(1)}$ conformal invariant theories. Commun. Math. Phys. 113, 1-26 (1987)

[9] Di Francesco, P.: Integrable lattice models, graphs and modular invariant conformal field theories. Int. J. Mod. Phys. A7, 407-500 (1992)

[10] Di Francesco, P., Zuber, J.-B.: $S U(N)$ lattice integrable models associated with graphs. Nucl. Phys. B338, 602-646 (1990)

[11] Di Francesco, P., Zuber, J.-B.: $S U(N)$ lattice integrable models and modular invariance. In: Recent Developments in Conformal Field Theories. Trieste 1989, Singapore: World Scientific 1990, pp. 179-215

[12] Dijkgraaf, R., Verlinde, E.: Modular invariance and the fusion algebras. Nucl. Phys. (Proc. Suppl.) 5B, 87-97 (1988)

[13] Doplicher, S., Haag, R., Roberts, J.E.: Fields, observables and gauge transformations. II. Commun. Math. Phys. 15, 173-200 (1969)

[14] Evans, D.E., Kawahigashi, Y.: Quantum symmetries on operator algebras. Oxford: Oxford University Press 1998

[15] Fredenhagen, K., Rehren, K.-H., Schroer, B.: Superselection sectors with braid group statistics and exchange algebras. II. Rev. Math. Phys. Special issue, 113-157 (1992)

[16] Fröhlich, J., Gabbiani, F.: Braid statistics in local quantum theory. Rev. Math. Phys. 2, 251-353 (1990)

[17] Fröhlich, J., Gabbiani, F.: Operator algebras and conformal field theory. Commun. Math. Phys. 155, 569-640 (1993)

[18] Fuchs, J., Schellekens, A.N., Schweigert, C.: Galois modular invariants of WZW models. Nucl. Phys. B437, 667-694 (1995)

[19] Gannon, T.: The classification of affine $S U(3)$ modular invariants. Commun. Math. Phys. 161, 233-264 (1994)

[20] Guido, D., Longo, R.: The conformal spin and statistics theorem. Commun. Math. Phys. 181, 11-35 (1996)

[21] Haag, R.: Local Quantum Physics. Berlin: Springer-Verlag 1992

[22] Izumi, M.: Subalgebras of infinite $C^{*}$-algebras with finite Watatani indices II: Cuntz-Krieger algebras. Duke Math. J. 91, 409-461 (1998) 
[23] Izumi, M., Longo, R., Popa, S.: A Galois correspondence for compact groups of automorphisms of von Neumann algebras with a generalization to Kac algebras. J. Funct. Anal. 155, 25-63 (1998)

[24] Jones, V.F.R.: Index for subfactors. Invent. Math. 72, 1-25 (1983)

[25] Kac, V.G.: Infinite dimensional Lie algebras, 3rd edition, Cambridge: Cambridge University Press, 1990

[26] Kato, A.: Classification of modular invariant partition functions in two dimensions. Mod. Phys. Lett. A2, 585-600 (1987)

[27] Longo, R.: Minimal index of braided subfactors. J. Funct. Anal. 109, 98-112 (1991)

[28] Longo, R., Rehren, K.-H.: Nets of subfactors. Rev. Math. Phys. 7, 567-597 (1995)

[29] Moore, G., Seiberg, N.: Naturality in conformal field theory. Nucl. Phys. B313, $16-40$ (1989)

[30] Ocneanu, A.: Paths on Coxeter diagrams: From Platonic solids and singularities to minimal models and subfactors. (Notes recorded by S. Goto.) In: Rajarama Bhat, B.V. et al. (eds.), Lectures on operator theory, The Fields Institute Monographs, Providence, Rhode Island: AMS publications 2000, pp. 243-323

[31] Petkova, V.B., Zuber, J.-B: From CFT to graphs. Nucl. Phys. B463, 161-193 (1996)

[32] Rehren, K.-H.: Braid group statistics and their superselection rules. In: Kastler, D. (ed.): The algebraic theory of superselection sectors. Palermo 1989, Singapore: World Scientific 1990, pp. 333-355

[33] Rehren, K.-H.: Space-time fields and exchange fields. Commun. Math. Phys. 132, 461-483 (1990)

[34] Rehren, K.-H.: Chiral observables and modular invariants. Commun. Math. Phys. 208, 689-712 (2000)

[35] Rehren, K.-H.: Canonical tensor product subfactors. Preprint hep-th/9911149

[36] Rehren, K.-H., Stanev, Y.S., Todorov, I.T.: Characterizing invariants for local extensions of current algebras. Commun. Math. Phys. 174, 605-633 (1996)

[37] Schellekens A.N., Yankielowicz, S.: Field identification fixed points in the coset construction. Nucl. Phys. B334, 67-102 (1990)

[38] Verlinde, E.: Fusion rules and modular transformations in 2D conformal field theory. Nucl. Phys. B300, 360-376 (1988)

[39] Verstegen, D.: New exceptional modular invariant partition functions for simple Kac-Moody algebras. Nucl. Phys. B346, 349-386 (1990)

[40] Wassermann, A.: Operator algebras and conformal field theory III: Fusion of positive energy representations of $L S U(N)$ using bounded operators. Invent. Math. 133, 467-538 (1998)

[41] Xu, F.: New braided endomorphisms from conformal inclusions. Commun. Math. Phys. 192, 347-403 (1998) 